\title{
Review \\ Complexity of the transcriptional network controlling secondary wall biosynthesis
}

\author{
Ruiqin Zhong and Zheng-Hua Ye \\ Department of Plant Biology, University of Georgia, Athens, GA 30602, USA
}

\footnotetext{
*Corresponding author:

Zheng-Hua Ye

Department of Plant Biology

University of Georgia

Athens, GA 30602

USA

Tel.: 1706542 1832; fax: 17065421805

E-mail: zhye@plantbio.uga.edu
} 


\section{ABSTRACT}

Secondary walls in the form of wood and fibers are the most abundant biomass produced by vascular plants, and are important raw materials for many industrial uses. Understanding how secondary walls are constructed is of significance in basic plant biology and also has far-reaching implications in genetic engineering of plant biomass better suited for various end uses, such as biofuel production. Secondary walls are composed of three major biopolymers, i.e., cellulose, hemicelluloses and lignin, the biosynthesis of which requires the coordinated transcriptional regulation of all their biosynthesis genes. Genomic and molecular studies have identified a number of transcription factors, whose expression is associated with secondary wall biosynthesis. We comprehensively review how these secondary wall-associated transcription factors function together to turn on the secondary wall biosynthetic program, which leads to secondary wall deposition in vascular plants. The transcriptional network regulating secondary wall biosynthesis employs a multi-leveled feed-forward loop regulatory structure, in which the top-level secondary wall NAC (NAM, ATAF1/2 and CUC2) master switches activate the second-level MYB master switches and they together induce the expression of downstream transcription factors and secondary wall biosynthesis genes. Secondary wall NAC master switches and secondary wall MYB master switches bind to and activate the SNBE (secondary wall NAC binding element) and SMRE (secondary wall MYB-responsive element) sites, respectively, in their target gene promoters. Further investigation of what and how developmental signals trigger the transcriptional network to regulate secondary wall biosynthesis and how different secondary wall-associated transcription factors function cooperatively in activating secondary wall biosynthetic pathways will lead to a better understanding of the molecular mechanisms underlying the transcriptional control of secondary wall biosynthesis. 
Keywords: Cell wall; MYB; NAC; Secondary wall; Transcription factor; Transcriptional regulation

\section{Contents}

1. Introduction

2. Secondary wall NAC master switches

2.1. Discovery of secondary wall NACs

2.2. Secondary wall NAC binding element

2.3. Direct targets of secondary wall NACs

2.4. Activators and repressors of secondary wall NACs

2.5. Evolutionary conservation of secondary wall NACs

3. Secondary wall MYB master switches

3.1. Discovery of secondary wall MYBs

3.2. Secondary wall MYB responsive element

3.3. Direct targets of secondary wall MYBs

4. Functions of NAC- and MYB-activated downstream transcription factors

5. Transcription factors regulating the lignin biosynthetic pathway

6. The model: feed-forward loop regulatory network

7. Coupled activation of secondary wall biosynthesis and programmed cell death

8. Concluding remarks

Acknowledgments

References 


\section{Introduction}

Secondary walls are deposited between the plasma membrane and the primary walls in specialized cells after the cessation of cell expansion. The most abundant cell types with secondary wall deposition are tracheary elements (tracheids in gymnosperms and vessels in angiosperms) and fibers in xylem/wood. Secondary walls provide mechanical strength and hydrophobicity for tracheids and vessels, facilitating their function as conduits of water transport. At the same time, the mechanical strength and rigidity conferred by secondary wall-reinforced tracheary elements and fibers provide structural support to plant organs, which enables vascular plants to reach great heights and compete for light. In addition to tracheary elements and fibers in xylem, secondary walls are also found in other cell types, such as sclereids in some fruits, seed coats and leaves, endothecium in anthers, valve margin fibers in seed pods, trichomes, and extraxylary fibers [1]. The ability for plants to deposit secondary walls was evolved when the first vascular plants evolved during the Silurian period around 430 million years ago. Thus, it is conceivable that plants might have co-opted the same regulatory mechanisms that activate the secondary wall biosynthetic program in various cell types. Dissecting the molecular mechanisms controlling secondary wall deposition would facilitate ascertaining the molecular signals and signaling pathways that initiate the program of secondary wall deposition, how the biosynthesis genes for secondary wall components are coordinately activated, and whether vascular plants evolved common regulatory mechanisms controlling secondary wall deposition in different cell types. Considering that secondary walls in the form of wood and fibers are the most abundant plant biomass widely used in our daily life, uncovering the molecular mechanisms controlling secondary wall deposition will also have important implications in tree biotechnology.

Secondary walls are mainly composed of cellulose, hemicelluloses and lignin, the proportions of which may vary in different cell types and plant species. All the biosynthesis 
genes for cellulose, hemicelluloses and lignin need to be turned on as well as the genes

responsible for the supply of nucleotide sugars, phenylpropanoid pathway precursors, methyl and acetyl donors and many other secondary wall biosynthetic pathway precursors need to be upregulated to make secondary walls. The secondary walls in tracheary elements are constructed in annular, helical, reticulated and pitted patterns. Thus, it is likely that specific genes responsible for the patterning of secondary walls are also induced. Genomic, coexpression and molecular analyses have provided ample evidence demonstrating the coordinated activation of genes involved in secondary wall biosynthesis during xylem and fiber differentiation $[2,3]$. In the past decade, comprehensive molecular and genetic studies have led to the breakthrough discovery that this coordinated activation of the secondary wall biosynthetic program is controlled by the secondary wall NAC (NAM, ATAF1/2 and CUC2)- and MYB-mediated transcriptional network (Fig. 1). This article will focus on recent findings on transcriptional regulation of secondary wall biosynthesis and attempt to postulate how different players in the transcriptional network function concertedly in the activation of secondary wall biosynthesis genes.

\section{Secondary wall NAC master switches}

\subsection{Discovery of secondary wall NACs}

Secondary wall NACs are master transcriptional switches controlling secondary wall deposition (Fig. 1; Table 1). They were first discovered during the study of xylem and fiber differentiation in the model plant Arabidopsis thaliana. Transcriptome profiling of Arabidopsis in vitro-induced xylem cells revealed the upregulation of 7 closely-related NAC domain genes, namely VASCULAR-RELATED NAC DOMAINs (VNDs), whose expression is specifically associated with vessel elements [3]. Overexpression of VND6 and VND7 in Arabidopsis induces ectopic deposition of vessel-like walls, and their dominant repression results in inhibition of 
metaxylem and protoxylem formation, respectively. This led to the conclusion that VNDs are transcriptional switches controlling protoxylem and metaxylem vessel formation. Similarly, VND1 to VND5 are specifically expressed in vessels and their overexpression results in ectopic deposition of secondary walls in normally parenchyma cells [4]. A role of NACs in regulating secondary wall thickening in anther endothecium was discovered by dominant repression of two Arabidopsis NAC genes, namely NAC SECONDARY WALL THICKENING PROMOTING FACTOR1 (NST1) and NST2, which results in an anther dehiscence defect [5]. NST1 and NST2 function redundantly in activating secondary wall biosynthesis in anther endothecium, and their overexpression induces the expression of secondary wall biosynthesis genes and concomitantly causes ectopic deposition of secondary walls in parenchymatous cells of various organs.

The most abundant secondary wall-forming cell types in Arabidopsis are fibers located in the xylem (xylary fibers) and outside the xylem (extraxylary fibers). Extraxylary fibers are present in various organs, such as the interfascicular regions (interfascicular fibers) of stems (Fig. 2A) [6] and the valve margin and the endocarp layer of siliques [7]. Studies of NAC genes that are preferentially expressed in developing stems led to the discovery of the role of SECONDARY WALL-ASSOCIATED NAC DOMAIN PROTEIN1 (SND1; also called NST3) in controlling secondary wall deposition in fibers $[6,8,9]$. The $S N D 1$ gene is specifically expressed in xylary fibers and interfascicular fibers but not in vessels in stems, and dominant repression of SND1 results in a specific loss of secondary wall thickening in fibers but not in vessels. Simultaneous RNA interference inhibition or loss-of-function mutations of SND1 and NST1 lead to a loss of secondary wall thickening in xylary fibers and interfascicular fibers of stems (Fig. 2B) and a pendent stem phenotype, demonstrating that SND1 functions redundantly with NST1 in activating the secondary wall biosynthetic program in fibers $[8,9]$. Simultaneous mutations of NST1 and SND1 also cause a loss of secondary wall thickening in endocarp and 
valve margin fibers of siliques, resulting in resistance to pod shattering [7]. In contrast, the resistance to pod shattering seen in domesticated soybeans was proposed to be due to an increased expression of a secondary wall NAC ortholog, SHAT1-5, which results in a significant secondary wall thickening of fiber cap cells in the pod [10]. Overexpression of SND1 activates the biosynthesis genes for cellulose, xylan and lignin, similar to NST1, NST2, and VND1 to VND7. This leads to ectopic deposition of secondary walls in leaf parenchyma cells (Fig. 2E) and concomitantly curly leaves and reduced plant growth [6]. These findings provide direct evidence that SND1 is a master transcriptional switch activating secondary wall biosynthesis in fibers.

Because VND1 to VND7, NST1, NST2 and SND1, which belong to the same subgroup in the Arabidopsis NAC family, are master transcriptional switches activating secondary wall biosynthesis albeit in different secondary wall-containing cell types, they have been named SECONDARY WALL NACs (SWNs) (Fig. 1) [11]. VND6 and VND7 were first proposed to control the differentiation of xylem cells based on the reports that their dominant repression specifically inhibits metaxylem and protoxylem vessel formation, respectively, and their overexpression results in ectopic deposition of patterned secondary walls [3]. However, similar to the effects of dominant repression or mutations of SND1 and NST1, which specifically affect the secondary wall thickening but not the cell identity of fiber cells $[8,9]$, dominant repression of VND6 and VND7 only results in a specific loss of secondary wall thickening in vessels but does not affect cell identity, i.e., the differentiation of vessels and their elongation are not affected [3]. Furthermore, although fiber secondary walls are uniformly thickened, the ectopically deposited secondary walls in the epidermal and mesophyll cells induced by overexpression of SND1 or NST1 exhibit helical and reticulated thickening patterns that are typically seen in xylem cells $[6,7,9]$. The patterned deposition of secondary walls is regulated by patterned organization of 
cortical microtubules and the ROP/MIDD1/Kinesin-13A complex [12]. These results indicate that different members of SWNs perform the same functions in regulating secondary wall biosynthesis, as evidenced by the finding that expression of VND6 or VND7 under the SND1 promoter rescues the secondary wall thickening defects in the snd Inst 1 double mutant.

\subsection{Secondary wall NAC binding element}

The finding that SWNs are master transcriptional switches of secondary wall biosynthesis provides an unprecedented tool to investigate how the secondary wall biosynthesis program is transcriptionally activated. SWNs regulate a number of downstream transcription factors (Fig. 1) $[6,13,14]$. Among them, MYB46 is a direct target and its promoter was used for mapping the SWN binding element [15]. Promoter deletion coupled with the electrophoretic mobility shift assay revealed that SWNs bind to an imperfect palindromic 19-bp consensus sequence, (T/A)NN(C/T)(T/C/G)TNNNNNNNA(A/C)GN(A/C/T)(A/T), which was named secondary wall NAC binding element (SNBE). Mutation of any of the conserved nucleotides in the SNBE sequence causes a loss of its binding by SWNs [15]. Since SWNs likely form homodimers or heterodimers [16], the arrangement of the palindromic sequence in SNBE may be required for its binding by the SWN dimers.

SNBEs are activated by all members of SWNs in transactivation analysis, albeit to different levels [15]. In addition, the SNBE sequences from the promoters of SWN direct target genes are sufficient to drive GUS reporter gene expression in the xylem and fiber cells in transgenic plants, demonstrating that the binding of the SNBE sequences by SWNs is critical for the tissues-specific expression of SWN direct genes $[15,17]$. The finding that all members of SWNs bind to and activate the SNBE sequences indicates that they are functional paralogs that regulate secondary wall biosynthesis in different cell types. This hypothesis is supported by 
complementation studies demonstrating that SWNs are functionally interchangeable $[15,18]$. Two other reports have also investigated the SWN binding elements. One study identified that the binding motif of SND1 is TACNTTNNNNATGA. The authors pointed out that most of the genes regulated by SND1 do not contain this motif [19], indicating that it is probably incorrect. Another report suggests that VND6 binds to the tracheary element-specific cis-element (namely TERE) composed of the core sequence CTTNAAAGCNA, which is present in the promoter of the XCP1 gene, a direct target of VND6 [20]. However, VND6, VND7 and SND1 did not bind to the TERE sequence [15] and the TERE sequences in the promoters of $X C P 1$ and $X C P 2$ were unable to drive the GUS reporter gene expression in xylem cells in transgenic plants [17]. On the contrary, the SNBE sequence in the $X C P 1$ promoter binds to and is activated by SWNs and is essential for the xylem-specific expression of $X C P 1$ [15]. Therefore, the available evidence does not support the TERE sequence as another binding element of SWNs, which is consistent with the observation that the majority of VND7 direct target genes do not contain TERE sequences in their promoters [18]. In contrast, SNBE sequences are present in the promoters of all known direct target genes of SWNs [15].

Although SWNs bind to and activate the SNBE sequences, they have different binding affinity toward different SNBE sequences, indicating that SWNs might differentially activate their target genes depending on which SNBE variants are present in the promoters of the target genes [15]. This hypothesis is congruent with the findings that SWNs, including SND1, VND6 and VND7, upregulate the expression of a common set of genes as well as unique ones $[15,18,20]$. Several genes involved in programmed cell death during xylem differentiation, including XCP1, XCP2, BFN1 and RNS3, are preferentially induced by VND6 and VND7 $[15,18,20]$ and the SNBE sequence from XCP1 is only activated in the xylem [17]. Several downstream transcription factors, such as LBD30, ANAC103, MYB55, IAA4, a SCARECROW 
family protein, a MYB transcription factor, a Dof-type zinc finger domain protein, an AP2domain transcription factor, and a basic helix-loop-helix protein, are specifically activated by either SND1 or VND7 [15]. These findings indicate that although SWNs are functionally interchangeable in terms of their activation of secondary wall deposition, they may have diverged and possess regulatory roles specific to the development of different cell types, such as vessels and fibers.

\subsection{Direct targets of secondary wall NACs}

SWNs induce the expression of a suite of transcription factors, including SND2, SND3, MYB46, MYB103, MYB85, MYB52, MYB54, MYB69, MYB42, MYB43, MYB20, and KNAT7, which led to the hypothesis that a transcriptional network encompassing SWNs and their downstream targets is involved in regulating secondary wall biosynthesis in secondary wallforming cells (Fig. 1) [6,13,14,21]. Among these SWN-regulated downstream transcription factors, $S N D 3, M Y B 46, M Y B 103$ and $K N A T 7$ are direct targets of SWNs as ascertained by direct target analysis [14]. Further global analyses of direct targets of SND1 and VND7 have revealed that SWNs not only directly activate downstream transcription factors but also directly induce the expression of genes involved in secondary wall biosynthesis, cell wall modification and programmed cell death $[15,18]$. This indicates the complexity of the transcriptional regulation of secondary wall biosynthesis. The direct targets of SWNs include additional transcription factors such as LOB domain proteins ( $L B D 15$ and $L B D 30$ ), $X N D 1$ and many other ones that have no known involvement in regulating secondary wall biosynthesis. The secondary wall biosynthesis genes directly induced by SWNs include several genes known to be involved in cellulose biosynthesis (CesA4 and CesA8), xylan biosynthesis (IRX14 homolog [22], IRX10 [23], PARVUS 
[24], UXS3 and UXS6 [25]) and a number of genes involved in monolignol transport and polymerization ( $\mathrm{ABC}$ transporters, peroxidases and laccases) [26-28].

A number of glycosyl hydrolase family genes are also directly activated by SWNs and they are probably involved in polysaccharide modifications during secondary wall biosynthesis and assembly. Another group of direct targets of SWNs are genes involved in cytoskeletal organization, vesicle transport and membrane fusion $[15,18]$. These include a microtubule depletion domain1 protein (MIDD1) that promotes local microtubule disassembly at the plasma membrane and thus prevents the cellulose synthase from depositing cellulose at pit areas of vessels [29], a RAC-like GTP-binding protein (ROP11) that in its activated form recruits MIDD1 to induce local disassembly of cortical microtubules for formation of vessel pits [30], several RAC GTPase activating proteins, a microtubule-associated protein (MAP65-8) that is likely to be involved in bundling of cortical microtubules during secondary wall formation [31], a syntaxin that is only activated by VND7 but not SND1, and several kinesin-like proteins that are probably involved in the transport of vesicles packed with secondary wall components and cellulose synthase complexes. One of these kinesin-like proteins is FRA1 that is required for normal secondary wall assembly and mechanical strength [32]. Other direct targets of SWNs include a MAP kinase, several LRR transmembrane protein kinases, and many hydrolases likely involved in programmed cell death. The functions of many other genes directly activated by SND1 and VND7 have not been characterized and they likely play important roles in secondary wall biosynthesis and assembly.

The promoters of all these direct targets of SWNs contain one or more SNBE sites, supporting the hypothesis that SWNs directly activate their expression via binding to the SNBE sites. This hypothesis is further substantiated by the promoter deletion analysis of two SWN direct targets, $M Y B 46$ and $X C P 1$, showing that the presence of the SNBE sites is directly 
correlated with the level of SWN-activated reporter gene expression [15]. The SNBE sites are not only present in the genes directly activated by SWNs but also in many other SWN-regulated downstream transcription factors and secondary wall biosynthesis genes that are not directly activated by SWNs. In particular, analysis of the $1.5-\mathrm{kb}$ promoter sequences of cellulose, xylan and lignin biosynthesis genes revealed the presence of SNBE sites in most of these genes (Fig. 3). However, most of these secondary wall genes are not directly induced by SWNs in direct activation analysis [15]. One hypothesis is that although SWNs alone are not sufficient to directly induce the expression of these secondary wall biosynthesis genes, their binding to the SNBE sites might be required for a combinatorial activation of the secondary wall biosynthesis genes with other transcription factors. The SNBE motif is highly degenerated and it is possible that some SNBE variants might have very low or no binding affinity to SWNs. Therefore, it is important to validate functional significance of predicted SNBE sites in a gene promoter using direct target analysis.

\subsection{Activators and repressors of secondary wall NACs}

Since SWNs are master transcriptional switches controlling secondary wall biosynthesis, the regulation of when, where and at what level they are expressed is a major determinant of secondary wall deposition in plants. Several transcription factors, including LBD18, LBD30, VNI2 and WRKY12 are activators or repressors of SWNs (Fig. 1; Table 1). Although it is currently unknown what regulators activate the tissue-specific expression of SWNs, SND1 appears to be able to bind to and activate its own promoter [19]. Overexpression of a poplar SWN homolog results in upregulation of other poplar SWN homologs [33,34]. The promoters of most of SWNs contain the SNBE sites (Fig. 3) and it is possible that some SWNs exert a positive feedback regulation on their own expression via binding to the SNBE sites. 
$L B D 18 / A S L 20$ and $L B D 30 / A S L 19$ are specifically expressed in xylem vessels in leaves and roots of young seedlings in Arabidopsis and their overexpression induces ectopic deposition of secondary walls [35]. Dominant repression of LBD18 leads to a reduction in the expression of $V N D 6$ and $V N D 7$ and a concomitant defect in secondary wall thickening in xylem vessel, whereas overexpression of LBD18 upregulates the expression of VND7. The expression of $L B D 18$ and $L B D 30$ is induced by VND6 and VND7 [35] and $L B D 30$ is a direct target of VND7 $[15,18]$. These findings indicate that LBD18 is involved in a positive feedback loop for the expression of VND6 and VND7.

Several transcription factors have been suggested as functioning as repressors that inhibit SWN function. One of these repressors is VNI2, a NAC domain protein that is able to interact with VND7 [36]. When coexpressed, VNI2 represses the VND7-mediated activation of the promoters of $L B D 30, M Y B 46$ and $X C P 1$. The repressor activity of VNI2 was confirmed by overexpression of VNI2, which resulted in a defect in secondary wall thickening in xylem vessel in leaves and roots of young seedlings and reduced plant growth. It was suggested that VNI2 acts as a transcriptional repressor by interacting with VNDs and thereby regulating their transcriptional activity. The expression of $V N I 2$ is not vessel specific and loss-of-function mutation of VNI2 has a major effect on leaf senescence but not on vessel formation [36,37]. In addition, some SWN homologs from poplar (Populus trichocarpa) and rice (Oryza sativa) have alternative splicing forms, leading to production of truncated SWNs without the C-terminal activation domain that may act as transcriptional repressors [34,38]. Overexpression of the Cterminal truncated form of a poplar SWN homolog, PtrSND1/PtrWND1B, represses the transactivation of target genes by its full-length form in protoplasts [34] and causes a reduction in fiber cell wall thickening in transgenic poplar wood and reduced plant growth [39]. Similarly, overexpression of C-terminal truncated VND7 driven by the VND7 promoter resulted in 
defective secondary wall thickening in vessels in transgenic Arabidopsis seedlings [16]. It is proposed that naturally occurring C-terminal truncated forms of SWNs might form dimers with SWNs to repress their transcriptional activities.

WRKY12 transcription factors might function as a repressor inhibiting secondary wall thickening in pith parenchyma cells [40]. Mutations of a Medicago WRKY12 gene results in ectopic deposition of secondary wall components in pith cells of stems and increased biomass production. In Arabidopsis, analysis of two T-DNA lines with insertions that occur in the same intron of the WRKY12 gene revealed ectopic deposition of secondary walls in wrky12-1 but not in wrky 12-2. Since the Arabidopsis wrky 12 mutant has an increased expression of NST2 and WRKY12 represses the NST2 promoter-driven reporter gene expression, it was suggested that WRKY12's function is to suppress the NST2-mediated activation of secondary wall thickening in the normally parenchymatous pith cells [40].

\subsection{Evolutionary conservation of secondary wall NACs}

SWN homologs are present in all taxa of land plants, including angiosperms, gymnosperms, primitive spore-bearing vascular plants (Selaginella moellendorffii) and nonvascular moss plants (Physcomitrella patens) [11] (Fig. 4). SWN homologs from a number of angiosperms, including poplar, Eucalyptus gunnii, spruce (Picea glauca), Medicago truncatula, rice, maize (Zea mays) and Brachypodium distachyon, are transcriptional regulators of secondary wall biosynthesis (Table 1). This suggests that the activation of secondary wall deposition by the SWN master transcriptional switches is a conserved mechanism in all vascular plants. The poplar genome has six pairs of SWN homologs (PtrWNDs/PtrVNSs) and each pair likely originated from its recent genome duplication. PtrWNDs are expressed in all wood-forming cells, although some of them are preferentially expressed in vessels or fibers [33,41-43]. Complementation 
analysis demonstrated that PtrWNDs are functional orthologs of Arabidopsis SWNs, as the expression of PtrWNDs rescues the secondary wall thickening defects in the fibers of the Arabidopsis sndlnst1 mutant. PtrWND overexpression in Arabidopsis and poplar activates the expression of secondary wall biosynthesis genes and induces ectopic deposition of secondary wall components, including cellulose, xylan and lignin [33,41,43]. Dominant repression of PtrWND functions results in defects in secondary wall thickening in vessels and fibers of woody tissues in transgenic poplar [33]. Similarly, a SWN homolog from Eucalyptus, EgWND1, is expressed in wood-forming cells of Eucalyptus stems, complements the secondary wall defects in the fibers of the sndlnst 1 mutant, and activates ectopic deposition of secondary walls when overexpressed in Arabidopsis [11]. Two SWN homologs from white spruce, $\operatorname{PgNAC-4}$ and $P g N A C-7$, activate the promoters of secondary wall biosynthesis genes [44]. These findings establish the essential roles of WNDs in regulating secondary wall biosynthesis during wood formation in tree species.

Multiple SWNs from monocotyledonous species, including rice [38;45], maize [45] and Brachypodium [46], have been identified and functionally characterized. Their overexpression in Arabidopsis activates secondary wall biosynthesis genes and concomitantly leading to ectopic deposition of secondary walls and reduced plant growth. Dominant repression of a rice SWN, OsSWN2, results in reduced secondary wall thickening in bundle sheath fibers of rice stems, stunted growth and wilting leaves [38]. Mutations of a Medicago SWN homolog, MtNST1, had a specific defect in secondary wall thickening of interfascicular fibers in stems and a concomitant reduction in the expression of secondary wall biosynthesis genes, demonstrating an important role of MtNST1 in regulating secondary wall biosynthesis in fibers [19,47]. SWNs from poplar, Eucalyptus, rice and maize are not only capable of functionally complementing the secondary wall defects in the fibers of the Arabidopsis snd1nst 1 mutant but also activate the expression of 
their direct target genes by binding to the SNBE sites. Together, these functional studies of SWN homologs from various species indicate that the $\mathrm{SWN}$-mediated transcriptional regulation of secondary wall biosynthesis is conserved in vascular plants. Since close homologs of SWNs are present in $P$. patens $[11,48]$, a nonvascular moss plant, it is likely that the first vascular plants coopted these preexisting ancestral NAC genes, via duplication and diversification, and function as secondary wall master transcriptional switches coordinating the expression of secondary wall biosynthesis genes in the first-evolved xylem conducting cells, tracheids [11]. These progenitor secondary wall NAC genes subsequently evolved as regulators of secondary wall biosynthesis in various secondary wall-forming cell types, including vessels and fibers in different taxa of vascular plants.

\section{Secondary wall MYB master switches}

\subsection{Discovery of secondary wall MYBs}

Studies of SWN-regulated downstream transcription factors in Arabidopsis have led to the discovery of second-level secondary wall master switches that are composed of two functionally redundant MYB proteins, MYB46 and MYB83. The MYB46 and MYB83 genes, which are direct targets of SWNs, are expressed in both vessels and fibers [13,49]. Their overexpression activates the expression of secondary wall biosynthesis genes for cellulose, xylan and lignin and concomitantly induces ectopic deposition of secondary wall components and reduced plant growth (Fig. 2F) [13,49,50]. Dominant repression or simultaneous RNA interference inhibition of MYB46 and MYB83 results in a drastic reduction in secondary wall thickening in both vessels and fibers, collapsed vessels and reduced plant growth (Fig. 2C) $[13,49]$, demonstrating their essential roles in regulating secondary wall thickening in both vessels and fibers. Single T-DNA knockout mutations of either MYB46 or MYB83 have no effect 
on secondary wall thickening, but simultaneous T-DNA knockout mutations of MYB46 and MYB83 led to a loss of secondary wall thickening in vessels with a severely collapsed vessel phenotype in young seedlings. As a result, the growth of the myb46 myb83 double mutant plants is arrested after germination, most likely due to lack of functional vessels for water transport [49]. These findings demonstrate that similar to SWNs, MYB46/83 are master switches capable of activating the entire secondary wall biosynthesis program. Since MYB46/83 are expressed in both vessels and fibers and are direct targets of all SWNs, it is evident that MYB46/83 act as the converging point for all SWNs in the SWN-mediated transcriptional network (Fig. 1).

MYB46/83 homologs have been identified and functionally characterized in a number of plant species, including gymnosperms (pine), dicot woody species (poplar and Eucalyptus) and monocots (rice and maize) (Fig. 5). These MYB46/83 homologs include PtMYB4/8 from pine [51,52], EgMYB2 from Eucalyptus [53], PtrMYB2/21 and PtrMYB3/20 from Populus trichocarpa [54,55], OsMYB46 from rice and ZmMYB46 from maize [45] (Table 1). All of them are expressed in secondary wall-forming cells, rescue the defective secondary wall phenotype when expressed under the MYB46 promoter in the myb46myb83 double mutant, and induce secondary wall biosynthesis genes and ectopic deposition of secondary walls when overexpressed [11,45,51-56]. Dominant repression of PtrMYBs results in a reduction in secondary wall thickening in the wood of transgenic poplar plants, which is consistent with their regulatory roles in activating secondary wall biosynthesis $[54,55]$. The available evidence indicates that similar to the top-level SWN master switches, the second-level MYB master switches are conserved in their regulation of secondary wall biosynthesis at least in gymnosperms and angiosperms. Although close MYB46/83 homologs are not present in the genome of spore-bearing vascular plants (Selaginella moellendorffii), it is possible that some less 
closely-related MYB homologs may function in place of MYB46/83 to activate secondary wall biosynthesis [11].

Another MYB transcription factor, MYB26, is essential for secondary wall thickening in anther endothecium of Arabidopsis [57,58]. MYB26 gene is expressed in the anther endothecial cells and its mutation results in a loss of secondary walls in the endothecium, leading to an anther indehiscence phenotype. Overexpression of MYB26 in both Arabidopsis and tobacco causes ectopic secondary wall thickening [58]. Based on the findings that MYB26 mutation reduces but its overexpression induces the expression of NST1/2, it was proposed that MYB26 functions upstream of NST1/2 in regulating secondary wall biosynthesis [58].

\subsection{Secondary wall MYB responsive element}

The DNA binding sequences of secondary wall MYBs were first investigated in pine and Eucalyptus MYBs (PtMYB4 and EgMYB2) [51,53]. These MYBs were originally thought to be specific regulators of lignin biosynthesis and therefore were tested for their ability to bind to and activate the AC elements (AC-I, ACCTACC; AC-II, ACCAACC; and AC-III, ACCTAAC), which are key cis-elements specifying the expression of lignin biosynthesis genes in xylem cells [59]. PtMYB4 and EgMYB2 bound and activated the AC elements, which led to the hypothesis that they regulate the expression of lignin biosynthesis genes via binding to the AC elements $[51,53]$. The finding that PtMYB4, EgMYB2 and Arabidopsis MYB46/83 are master switches of the entire secondary wall biosynthetic program suggests that they may bind to additional cis elements in addition to the AC elements. A 7-bp sequence, designated as the secondary wall MYB-responsive element (SMRE), is sufficient to be responsive to MYB46 activation as ascertained using promoter deletion coupled with transactivation analysis of a MYB46 direct target. Mapping of the consensus sequence revealed that MYB46 and MYB83 are able to bind to 
and activate all the eight variants of SMRE, $\mathrm{ACC}(\mathrm{A} / \mathrm{T}) \mathrm{A}(\mathrm{A} / \mathrm{C})(\mathrm{T} / \mathrm{C})[60]$. Using a similar approach, an eight nucleotide core motif, $(\mathrm{T} / \mathrm{C}) \mathrm{ACC}(\mathrm{A} / \mathrm{T}) \mathrm{A}(\mathrm{A} / \mathrm{C})(\mathrm{T} / \mathrm{C})$, was identified as the MYB46-responsive cis-regulatory element (M46RE) [61]. This M46RE site differs from the SMRE site by having one additional nucleotide at one end of the sequence, indicating that the two separate studies led to identification of the same core cis-element for MYB46/83 binding. The promoter of MYB54, which is a direct target of MYB46, has 2 SMRE sites [60] but no M46RE sites [61], suggesting that the extra nucleotide in the M46RE site is not required for MYB46/83 activation and thereby the SMRE site represents the MYB46/83 binding motif. Three of the eight SMRE variants are identical with the AC elements (SMRE8/AC-I, ACCTACC; SMRE4/AC-II, ACCAACC; and SMRE7/AC-III, ACCTAAC), which is consistent with the previous reports that the pine and Eucalyptus MYB46/83 homologs, PtMYB4 and EgMYB2, bind to the AC elements [51,53]. PtMYB4 and EgMYB2 bind to and activate the other five SMRE variants in addition to the AC elements [55]. Poplar MYB46/83 homologs, PtrMYB2/21 and PtrMYB3/20 also bind to and activate the SMRE sites [55]. These findings are congruent with the complementation analysis showing that the pine, Eucalyptus and poplar MYB46/83 homologs are all capable of rescuing the secondary wall defects in the Arabidopsis myb46myb83 double mutant. The finding that MYB46/83 and their orthologs from gymnosperms and angiosperms all bind to and activate the SMRE sites provides additional line of evidence supporting the hypothesis that the MYB46/83-like secondary wall MYBs act as second-level master switches regulating secondary wall biosynthesis in vascular plants.

The eight SMRE variants have differential responses to the activation by MYB46/83 and their homologs from pine, Eucalyptus and poplar [55,60]. Among them, SMRE5 and SMRE7 have the highest activation by MYB46/83. This indicates that the expression level of genes 
directly induced by MYB46/83 may largely depend on the presence of the particular SMRE variants in the target gene promoters.

\subsection{Direct targets of secondary wall MYBs}

MYB46/83 and their orthologs not only activate secondary wall biosynthesis genes but also induce the expression of a group of transcription factors [13,49,50,55,60,62]. Among the MYB46/83-regulated downstream transcription factors, MYB4, MYB6, MYB7, MYB32, MYB43, MYB52, MYB54, MYB58, MYB63, KNAT7, BEL10, bZIP6, TRY, IAA28, BLH2, BLH3, BLH6, AtC3H14, and ZAT5 are direct targets of MYB46/83 [60]. A plethora of genes involved in polysaccharide and lignin biosynthesis, cytoskeletal organization, signaling, cell wall modification, and programmed cell death are other direct targets of MYB46/83 [60,63,64]. The promoters of all these MYB46 direct target genes contain one or more SMRE sites, consistent with the hypothesis that MYB46 directly regulates the expression of its direct targets via binding to the SMRE sites. These findings demonstrate that similar to the top-level SWN master switches, the second-level MYB46/83 master switches directly activate not only downstream transcription factors but also genes involved in the biosynthesis of secondary wall components. However, some of secondary wall biosynthesis genes are not directly induced by MYB46 although the promoters of most of them contain the SMRE sites (Fig. 3). It is possible that even though MYB46/83 could bind to these SMRE sites in vivo, their binding alone is insufficient to

directly activate the expression of these secondary wall biosynthesis genes. MYB46/83 probably act in concert with SWNs and other transcription factors, achieving the maximum level of induction of secondary wall biosynthesis genes.

\section{Functions of NAC- and MYB-activated downstream transcription factors}


The SWN and MYB46/83 master switches activate a battery of downstream transcription factors. Two Arabidopsis NAC genes, SND2 and SND3, which are induced by SWNs, are specifically expressed in secondary wall-forming cells. When their functions are dominantly repressed in transgenic plants, the secondary wall thickening in fibers is inhibited. When overexpressed, they activate a number of secondary wall biosynthesis genes, resulting in increased secondary wall thickening in fibers [14,65]. The poplar SND2 orthologs (PtrNAC154 and PtrNAC156) and SND3 orthologs (PtrNAC105 and PtrNAC157) are also downstream targets regulated by poplar SWN orthologs (PtrWNDs) and are expressed in secondary xylem cells during wood formation $[33,66]$. Dominant repression of the function of PtrNAC154 (PtSND2) causes reduced expression of secondary wall biosynthesis genes, thinner xylary fibers and reduced plant growth [67]. SND2 and SND3 are important regulators of secondary wall biosynthesis, but their overexpression is insufficient to induce ectopic deposition of secondary walls. The available evidence indicates that SND2 and SND3 may function cooperatively with other transcription factors in activating secondary wall biosynthesis genes.

The secondary wall-associated homeodomain protein KNAT7 is a direct target of both SWNs and MYB46/83 [14,49,60,61]. Although KNAT7 overexpression does not lead to activation of secondary wall biosynthesis genes, its mutation or dominant repression resulted in a collapsed vessel phenotype and reduced secondary wall thickening $[14,68]$. KNAT7 was proposed to be a transcriptional repressor that negatively regulates secondary wall biosynthesis based on the observation that the knat7 mutant has the increased secondary wall thickening in fibers, which is opposite to the reduced secondary wall thickening seen in the vessels of the knat7 mutant [69]. Furthermore, KNAT7 interacts with MYB75 and an OVATE FAMILY PROTEIN, OFP4, and it was hypothesized that KNAT7 forms a functional complex with the MYB75 and OFP proteins to exert its regulation of secondary wall biosynthesis [70,71]. 
A zinc finger protein, AtC3H14, whose expression is induced by MYB46, was proposed to be another master regulator of secondary wall biosynthesis. This is based on the transactivation analysis in protoplasts showing that it activates reporter gene expression driven by the promoters of several biosynthesis genes for cellulose, xylan and lignin [50]. AtC3H14 is localized in specialized cytoplasmic processing bodies instead of the nucleus [72]. Thus, it is unlikely that it functions as a transcription factor.

Additional SWN- and MYB46/83-regulated downstream transcription factors include LOB15, LOB30, MYB4, MYB7, MYB20, MYB32, MYB42, MYB43, MYB52, MYB54, MYB58, MYB63, MYB69, MYB85, MYB103, BEL10, bZIP6, TRY, IAA28, BLH2, BLH3, BLH6, HB5, HB15 and XND1 [6,13-15,18,20,50,60,62]. As previously discussed, LOB30 has a role in regulating secondary wall biosynthesis in vessels and is probably involved in a positive feedback loop that regulates VND7 expression [35]. XND1, a direct target of SWNs [15], is a NAC protein that is proposed to negatively regulate secondary wall biosynthesis and programmed cell death based on the observation that its overexpression results in extreme dwarfism associated with the absence of xylem cells and little expression of xylem marker genes [73]. The MYB52, MYB54, MYB69, and MYB103 genes are specifically expressed in secondary wall-forming cells and their dominant repression results in a reduction in secondary wall thickening in fiber cells, indicating that they are involved in regulation of secondary wall biosynthesis [14]. MYB4, MYB7, MYB32, MYB58, MYB63, MYB85, BLH6, HB5 and HB15 are implicated in regulating lignin biosynthesis and will be discussed below. Other SWN- and MYB46/83-regulated transcription factors have not been characterized for their roles in transcriptional regulation of secondary wall biosynthesis.

\section{Transcription factors regulating the lignin biosynthesis pathway}


A number of transcriptional activators and repressors regulate the expression of genes involved in lignin biosynthesis. Two closely related Arabidopsis MYBs, MYB58 and MYB63, which are direct targets of MYB46/83 [60], are specific transcriptional activators of lignin biosynthesis [74]. They are specifically expressed in secondary wall-forming cells and their dominant repression leads to a reduction in secondary wall thickening and lignin content and weakened mechanical strength of stems, whereas their overexpression induces the expression of lignin biosynthesis genes, ectopic deposition of lignin but not cellulose and xylan, and reduced plant growth. MYB58 and MYB63 directly activate the expression of lignin biosynthesis genes via binding to the AC elements in their promoters. Two poplar MYB58/63 orthologs, PtrMYB28 and PtrMYB192, are induced by PtrWNDs [33] and PtrMYB28 regulates the expression of lignin biosynthesis genes in an AC element-dependent manner [56].

Other transcriptional activators of lignin biosynthesis include MYB85 and its pine ortholog PtMYB1, whose overexpression results in ectopic deposition of lignin [14,52], a poplar MYB43 ortholog, PtrMYB152, whose overexpression results in increased lignin production [75], and MYB103 which was proposed to specifically regulate the expression of ferulate 5hydroxylase and the production of syringyl lignin [76]. A tobacco LIM family protein, NtLIM1, activates the AC element-driven GUS reporter gene and antisense inhibition of its expression results in reduced lignin content in tobacco stems [77]. An earlier study reported that SND1 directly regulates the expression of a Medicago ferulate 5-hydroxylase $(F 5 H)$ gene and the production of syringyl lignin [78]. In Arabidopsis, SND1 expression alone is insufficient to directly activate the expression of $F 5 H[15,76]$.

The Arabidopsis MYB61, which binds to a six-nucleotide cis element, ACC(A/T)A(A/C), regulates the expression of a specific set of target genes, including a caffeoyl CoA $O$-methyltransferase (CCoAOMT7) and KNAT7 [79]. MYB61 overexpression results in 
ectopic deposition of lignin, implicating its role in regulating lignin biosynthesis [80]. Mutational analysis of several genes whose expression is induced by overexpression of EgMYB2 in Arabidopsis revealed additional genes that might be involved in regulating lignin biosynthesis [62]. An Arabidopsis mutant with mutations in a homeodomain-containing protein, BLH6, which is a direct target of MYB46 [60], has reduced lignification in fiber cell walls [62]. The rice BLH6 ortholog is also associated with secondary wall biosynthesis as its overexpression in transgenic rice leads to ectopic deposition of lignin [81].

MYB4, MYB7 and MYB32 and their orthologs in maize, Eucalyptus, and switchgrass have been proposed to be transcriptional repressors of lignin biosynthesis. Mutations of MYB4 and MYB32 lead to an induction of expression of a few lignin biosynthesis genes $[82,83]$, and overexpression of their orthologs from maize (ZmMYB31 and ZmMYB42), Eucalyptus (EgMYB1) and switchgrass (PvMYB4) results in downregulation of expression of lignin biosynthesis genes and a reduction in lignin content [84-88]. EgMYB1 overexpression in poplar and Arabidopsis also results in downregulation of the expression of biosynthesis genes for cellulose and xylan. Therefore, EgMYB1 was proposed to be a general repressor of secondary wall biosynthesis [85]. EgMYB1 was expressed in developing xylem where lignification occurs [84], whereas the association of the expression of other MYB repressors with secondary wallforming cells has not been demonstrated. Since these MYB repressors bind to AC elements [87,88], they might compete with the MYB activators for binding to AC elements, resulting in the repression of lignin biosynthesis. Several other transcription factors, including MYB52, another MYB-like transcription factor (At3g11280), HB5 and HB15 are also suggested to be negative regulators of lignin biosynthesis based on the observations that their mutations result in hyperlignified secondary cell walls [62]. 


\section{Transcription factors associated with wood formation in tree species}

Studies of transcriptional regulation of wood formation in poplar have revealed that the transcriptional network regulating secondary wall biosynthesis is conserved among herbaceous Arabidopsis and woody tree species $[33,41,43,54,55]$. Similar to Arabidopsis, poplar also utilizes secondary wall NAC transcription factors, PtrWNDs, as the top-level master switches and MYB46/83 orthologs, PtrMYB2/3/20/21, as the second-level master switches (Fig. 1). These poplar NAC and MYB master switches activate an array of downstream transcription factors, including those that are orthologs of Arabidopsis SWN- and MYB46/83-regulated downstream transcription factors [33]. Since wood formation requires a massive deposition of secondary walls, it is likely that poplar might have evolved additional key transcriptional regulators that sustain a high-level activation of secondary wall biosynthesis genes during wood formation. Indeed, PtrWND master switches activate a set of additional downstream transcription factors, whose Arabidopsis counterparts are not known to be induced by SWNs $[33,89]$. Furthermore, in addition to the known second-level MYB master switches (PtrMYB2/3/20/21), a number of other PtrWND-regulated transcription factors, including PtrMYB18, PtrMYB74, PtrMYB75, PtrMYB121, PtrMYB128, PtrZF1, PtrGATA8, PtrNAC150, PtrNAC156 and PtrNAC157, are also capable of activating the promoters of biosynthesis genes for cellulose, xylan and lignin, suggesting that they might also function as key regulators of secondary wall biosynthesis [33].

\section{The model: feed-forward loop regulatory network}

Based on the early studies of SWNs and MYB46/83, it appeared that the transcriptional network regulating secondary wall biosynthesis involves a linear cascade in which the top-level SWN master switches activate the second-level MYB master switches, which further activate downstream transcription factors, thus leading to the activation of the secondary wall 
biosynthesis program. However, studies of direct targets and DNA binding elements of SWNs and MYB46/83 led to the discovery that they directly activate not only downstream transcription factors but also a number of secondary wall biosynthesis genes (Fig. 1). Of particular interest is that SWNs and their direct target MYB46/83 both directly activate a set of common target genes, such as the transcription factor KNAT7 and several biosynthesis genes for xylan and lignin. Similarly, MYB46/83 and their direct target MYB58/63 and MYB4/7/32 all directly regulate the expression of lignin biosynthesis genes $[15,60]$. This type of regulatory structure in which one transcription factor regulates another and they together regulate downstream targets is coined as a feed-forward loop regulatory network. It is apparent that vascular plants evolved a multipleleveled feed-forward loop regulatory structure that ensures the efficient activation of secondary wall biosynthesis genes. Because the promoters of most of the secondary wall biosynthesis genes contain the DNA binding elements of SWNs and MYB46/83 (Fig. 3), it is tempting to propose that both SWNs and MYB46/83 bind to the promoters of these secondary wall biosynthesis genes. It is likely that SWNs, MYB46/83 and their downstream transcription factors function concertedly to bind to and activate the promoters of secondary wall biosynthesis genes for their maximal expression (Fig. 1).

\section{Coupled activation of secondary wall biosynthesis and programmed cell death}

In addition to downstream transcription factors and secondary wall biosynthesis genes, SWNs also activate the expression of genes involved in cell wall modification and programmed cell death $[15,18,20,46]$. A number of glycosyl hydrolase genes are direct targets of SWNs [15] and some of them are close homologs of poplar genes associated with wood formation [90]. This indicates that they might be involved in cell wall modification during secondary wall thickening. Programmed cell death-related hydrolases activated by SWNs include nucleases (RNS3 and 
$\mathrm{BFN} 1$ ), cysteine proteases (XCP1 and $\mathrm{XCP} 2)$, serine proteases, an aspartyl protease, metacaspases and lipases. Some of these nucleases (BFN1) and proteases (XCP1 and XCP2) have been suggested to be involved in the degradation of cellular contents during the maturation of tracheary elements $[91,92]$. The promoters of all these genes contain multiple SNBE sites and in the case of XCP1, two SNBE sites have been confirmed to be responsible for the SWNmediated transcriptional activation [15]. This indicates that these programmed cell death-related genes are directly activated via binding of the SNBE sites by SWNs. The vessel-specific SWNs (VND6 and VND7) are stronger activators of programmed cell death genes than fiber-specific SWNs. This is consistent with the fact that vessels undergo programmed cell death immediately following secondary wall thickening. Fiber cells undergo a different mode of programmed cell death [93] and different hydrolase genes might be involved in this process. These findings indicate that vascular plants evolved SWNs as regulators of the expression of genes controlling secondary wall thickening and programmed cell death during tracheary element development. In addition to SWNs, MYB46 also directly activates the expression of several cysteine protease and serine protease genes, underlining its role in regulating programmed cell death [60].

The ability of SWNs to regulate programmed cell death might be directly co-opted from SWN progenitors in non-vascular plants. Close homologs of SWNs in moss regulate programmed cell death in water-conducting cells [48]. Mosses do not have secondary wall thickening and the expression of SWN homologs is not correlated with the location of xylans in mosses [94]. This indicates that moss SWN homologs may not regulate genes related to the biosynthesis of secondary wall components. One tentative hypothesis is that vascular plants coopted SWN progenitors from non-vascular plants and they evolved new functions, i.e., regulation of secondary wall biosynthesis and cell wall modification, in addition to their primitive functions in regulation of programmed cell death. Three Arabidopsis root cap cell-specific NACs, 
SOMBRERO (SMB), BEARSKIN1 (BRN1) and BRN2, which are closely related to SWNs, are required for root cap cell maturation [95]. Although ectopic expression of these NACs can induce secondary wall deposition, they are not involved in controlling secondary wall biosynthesis in root cap cells since these cells do not contain secondary walls. Mutations of these NACs led to downregulation of the expression of several cellulase genes and programmed cell death-related genes and concomitantly, a failure of programmed cell death and detachment of root cap cells $[95,96]$. These findings indicate that in contrast to SWNs, SMB/BRN1/BRN2 evolved to specifically regulate cell wall modification and programmed cell death during root cap development.

\section{Concluding remarks}

Secondary wall biosynthesis is a complex process involving the activation of many genes required for precursor supply, biosynthesis of secondary wall components, and patterned wall deposition. Molecular and genetic analyses of secondary wall biosynthesis in Arabidopsis have uncovered a feed-forward loop transcriptional regulatory network controlling the activation of the secondary wall biosynthetic program (Fig. 1). The top-level SWN master switches are present in all vascular plants and they regulate secondary wall biosynthesis, indicating the evolutionary conservation of the secondary wall transcriptional network in vascular plants [11]. Because the genome of the non-vascular moss $P$. patens harbors SWN-like NAC genes, it was proposed that SWN progenitors in the first vascular plants evolved new functions, i.e., transcriptional regulation of secondary wall biosynthesis in tracheids, the first-evolved waterconducting cells [11]. During the evolution of diverse taxa of vascular plants, SWNs evolved as regulators of secondary wall biosynthesis in fibers and other sclerenchymatous cells. The second-level MYB master switches appear to be conserved at least in gymnosperms and 
angiosperms because MYB46/83-like MYBs from pine, poplar, rice and maize are all functional orthologs of Arabidopsis MYB46/83 in regulating secondary wall biosynthesis. However, secondary walls in different species or in various cell types within the same species differ in their composition, indicating that the degree of transcriptional activation of the biosynthetic pathways for individual secondary wall components may vary. It is likely that downstream transcription factors regulated by NAC and MYB master switches have important roles in enhancing or repressing the biosynthesis genes of different secondary wall components. This may result in variations of secondary wall composition in different species and different cell types within the same species. The exact functions of many of the SWN- and MYB46/83-regulated transcription factors are currently unclear, and further studies of their functions will provide insight into how they act together with other transcription factors and regulate secondary wall biosynthesis.

Differentiation of secondary wall-forming cells is a sequential developmental process that involves initiation of cell differentiation, cell expansion, secondary wall thickening and in the case of tracheary elements, programmed cell death. Because secondary wall thickening occurs after cessation of cell expansion in secondary wall-forming cell types, it is reasonable to propose that after cessation of cell expansion, cells generate signals that induce the expression of SWNs, which ultimately lead to activation of the secondary wall biosynthetic program. It will be of immense importance to find what signals and signal transduction pathways trigger the activation of the transcriptional network leading to secondary wall biosynthesis in secondary wall-forming cells. Because secondary walls produced by vascular plants represent the bulk of plant biomass targeted for production of lignocellulosic biofuels, a complete understanding of how secondary walls are produced will provide valuable tools for custom-designing wall components tailored for biofuel production. An example of custom-designing wall components is to increase 
polysaccharide deposition using SWNs in a lignin-deficient mutant [97], which could potentially result in higher sugar yield in biomass feedstock for biofuel production.

\section{Acknowledgments}

The work in the authors' lab was funded by the US Department of Agriculture National Institute of Food and Agriculture [AFRI Plant Biology program (\#2010-65116-20468)], the National Science Foundation (ISO-1051900) and the Division of Chemical Sciences, Geosciences, and Biosciences, Office of Basic Energy Sciences of the U.S. Department of Energy (DE-FG02-03ER15415).

\section{References}

[1] J.D. Mauseth, Plant Anatomy. The Benjamin/Cummings Publishing Company, Inc, Menlo Park, California, 1988.

[2] T. Demura, et al., Visualization by comprehensive microarray analysis of gene expression programs during transdifferentiation of mesophyll cells into xylem cells, Proc. Natl. Acad. Sci. USA 99 (2002) 15794-15799.

[3] M. Kubo, et al., Transcription switches for protoxylem and metaxylem vessel formation, Genes Dev. 19 (2005) 1855-1860.

[4] J. Zhou, R. Zhong, Z.-H. Ye. Arabidopsis NAC domain proteins, VND1 to VND5, are transcriptional regulators of secondary wall biosynthesis in vessels. PLoS One 9 (2014) e105726.

[5] N. Mitsuda, M. Seki, K. Shinozaki, M. Ohme-Takagi, The NAC transcription factors NST1 and NST2 of Arabidopsis regulates secondary wall thickening and are required for anther dehiscence. Plant Cell 17 (2005) 2993-3006. 
[6] R. Zhong, T. Demura, Z.-H. Ye, SND1, a NAC domain transcription factor, is a key regulator of secondary wall synthesis in fibers of Arabidopsis, Plant Cell 18 (2006) 3158-3170.

[7] N. Mitsuda, M. Ohme-Takagi, NAC transcription factors NST1 and NST3 regulate pod shattering in a partially redundant manner by promoting secondary wall formation after the establishment of tissue identity. Plant J. 56 (2008) 768-778.

[8] R. Zhong, E.A. Richardson, Z.-H. Ye, Two NAC domain transcription factors, SND1 and NST1, function redundantly in regulation of secondary wall synthesis in fibers of Arabidopsis, Planta 225 (2007) 1603-1611.

[9] N. Mitsuda, et al., NAC transcription factors, NST1 and NST3, are key regulators of the formation of secondary walls in woody tissues of Arabidopsis, Plant Cell 19 (2007) 270-280. [10] Y. Dong, et al., Pod shattering resistance associated with domestication is mediated by a NAC gene in soybean. Nat. Commun. 5 (2014) 3352.

[11] R. Zhong, C. Lee,, Z.-H. Ye, Evolutionary conservation of the transcriptional network regulating secondary cell wall biosynthesis. Trends Plant Sci. 15 (2010) 625-631.

[12] Y. Oda, H. Fukuda, Secondary cell wall patterning during xylem differentiation. Curr. Opin. Plant Biol. 15 (2012) 38-44.

[13] R. Zhong, E.A. Richardson, Z.-H. Ye, The MYB46 transcription factor is a direct target of SND1 and regulates secondary wall biosynthesis in Arabidopsis, Plant Cell 19 (2007) 27762792.

[14] R. Zhong, C. Lee, J. Zhou, R.L. McCarthy, Z.-H. Ye, A battery of transcription factors involved in the regulation of secondary cell wall biosynthesis in Arabidopsis, Plant Cell 20 (2008) 2763-2782.

[15] R. Zhong, C. Lee, Z.-H. Ye, Global analysis of direct targets of secondary wall NAC master switches in Arabidopsis, Mol. Plant 3 (2010) 1087-1103. 
[16] M. Yamaguchi, M. Kubo, H. Fukuda, T. Demura, Vascular-related NAC-DOMAIN7 is involved in the differentiation of all types of xylem vessels in Arabidopsis roots and shoots, Plant J. 55 (2008) 652-664.

[17] R.L. McCarthy, R. Zhong, Z.-H. Ye, Secondary wall NAC binding element (SNBE), a key cis-acting element required for target gene activation by secondary wall NAC master switches, Plant Signal Behav. 6 (2011) 1282-1285.

[18] M. Yamaguchi, et al., VASCULAR-RELATED NAC-DOMAIN7 directly regulates the expression of a broad range of genes for xylem vessel formation, Plant J. 66 (2011) 579-590. [19] H. Wang, Q. Zhao, F. Chen, M. Wang, R.A. Dixon, NAC domain function and transcriptional control of a secondary cell wall master switch, Plant J. 68 (2011) 1104-1114. [20] K. Ohashi-Ito, Y. Oda, H. Fukuda, Arabidopsis VASCULAR-RELATED NAC-DOMAIN6 directly regulates the genes that govern programmed cell death and secondary wall formation during xylem differentiation, Plant Cell 22 (2010) 3461-3473.

[21] R. Zhong, Z.-H. Ye, Regulation of cell wall biosynthesis. Curr. Opin. Plant Biol. 10 (2007) 564-572.

[22] C. Lee, Q. Teng, W. Huang, R. Zhong, Z.-H. Ye, The Arabidopsis family GT43 glycosyltransferases form two functionally nonredundant groups essential for the elongation of glucuronoxylan backbone, Plant Physiol. 153 (2010) 526-541.

[23] D.M. Brown, Z. Zhang, E. Stephens, P. Dupree, S.R. Turner, Characterization of IRX10 and IRX10-like reveals an essential role in glucuronoxylan biosynthesis in Arabidopsis, Plant J. 57 (2009) 732-746.

[24] C. Lee, et al., The PARVUS gene is expressed in cells undergoing secondary wall thickening and is essential for glucuronoxylan biosynthesis, Plant Cell Physiol. 48 (2007b) 1659-1672. 
[25] S. Pattathil, A.D. Harper, M.Bar-Peled, Biosynthesis of UDP-xylose: characterization of membrane-bound AtUxs2. Planta 221 (2005) 538-548.

[26] S. Berthet, et al., Disruption of LACCASE4 and 17 results in tissue-specific alterations to lignification of Arabidopsis thaliana stems, Plant Cell 23 (2011) 1124-1137.

[27] S. Alejandro, et al., AtABCG29 is a monolignol transporter involved in lignin biosynthesis, Curr. Biol. 22 (2012) 1207-1212.

[28] J. Herrero, A. Esteban-Carrasco, J.M. Zapata JM, Looking for Arabidopsis thaliana peroxidases involved in lignin biosynthesis, Plant Physiol. Biochem. 67 (2013) 77-86. [29] Y. Oda, Y. Iida, Y. Kondo, H. Fukuda, Wood cell-wall structure requires local 2Dmicrotubule disassembly by a novel plasma membrane-anchored protein. Curr. Biol. 20 (2010) 1197-1202.

[30] Y. Oda, H. Fukuda, Initiation of cell wall pattern by a Rho- and microtubule-driven symmetry breaking. Science 337 (2012) 1333-1336.

[31] G. Mao, H. Buschmann, J.H. Doonan, CW. Lloyd, The role of MAP65-1 in microtubule bundling during Zinnia tracheary element formation. J. Cell Sci. 119 (2006) 753-758. [32] R. Zhong, D.H. Burk, W.H.III Morrison, Z.-H. Ye, A Kinesin-like protein is essential for oriented deposition of cellulose microfibrils and cell wall strength. Plant Cell 14 (2002) 31013117.

[33] R. Zhong, R.L. McCarthy, C. Lee, Z.-H. Ye, Dissection of the transcriptional program regulating secondary wall biosynthesis during wood formation in poplar, Plant Physiol. 157 (2011) 1452-1468.

[34] Q. Li, et al., Splice variant of the SND1 transcription factor is a dominant negative of SND1 members and their regulation in Populus trichocarpa, Proc. Natl. Acad. Sci. USA 109 (2012) 14699-14704. 
[35] T, Soyano, S. Thitamadee, Y. Machida, N.H. Chua, ASYMMETRIC LEAVES2-

LIKE19/LATERAL ORGAN BOUNDARIES DOMAIN30 and ASL20/LBD18 regulate tracheary element differentiation in Arabidopsis, Plant Cell. 20 (2008) 3359-3373.

[36] M. Yamaguchi, et al., VND-INTERACTING2, a NAC domain transcription factor, negatively regulates xylem vessel formation in Arabidopsis, Plant Cell 22 (2010) 1249-1263. [37] S.D. Yang, P.J. Seo, H.K. Yoon, C.M. Park, The Arabidopsis NAC transcription factor VNI2 integrates abscisic acid signals into leaf senescence via the COR/RD genes. Plant Cell 23 (2011) 2155-2168.

[38] K. Yoshida, S. Sakamoto, T. Kawai, Y. Kobayashi, K. Sato, Y. Ichinose, K. Yaoi, M. Akiyoshi-Endo, H. Sato, T. Takamizo, M. Ohme-Takagi, N. Mitsuda, Engineering the Oryza sativa cell wall with rice NAC transcription factors regulating secondary wall formation. Front. Plant Sci. 4 (2013) 383.

[39] Y. Zhao, J. Sun, P. Xu, R. Zhang, L. Li, Intron-mediated alternative splicing of WOODASSOCIATED NAC TRANSCRIPTION FACTOR1B regulates cell wall thickening during fiber development in Populus species. Plant Physiol. 164 (2014) 765-776.

[40] H. Wang, et al., Mutation of WRKY transcription factors initiates pith secondary wall formation and increases stem biomass in dicotyledonous plants. Proc. Natl. Acad. Sci. USA 107 (2010) 22338-22343.

[41] R. Zhong, C. Lee, Z.-H. Ye, Functional characterization of poplar wood-associated NAC domain transcription factors, Plant Physiol. 152 (2010) 1044-1055.

[42] R. Zhong, Z.-H. Ye, The poplar PtrWNDs are transcriptional activators of secondary cell wall biosynthesis, Plant Signal. Behav. 5 (2010) 469-472.

[43] M. Ohtani, et al., A NAC domain protein family contributing to the regulation of wood formation in poplar. Plant J. 67 (2011) 499-512. 
[44] I. Duval, et al., Large-scale screening of transcription factor-promoter interactions in spruce reveals a transcriptional network involved in vascular development. J. Exp. Bot. 65 (2014) 23192333.

[45] R. Zhong, et al., Transcriptional activation of secondary wall biosynthesis by rice and maize NAC and MYB transcription factors, Plant Cell Physiol. 52 (2011a) 1856-1871.

[46] E.R. Valdivia, et al., Regulation of secondary wall synthesis and cell death by NAC transcription factors in the monocot Brachypodium distachyon, J. Exp. Bot. 64 (2013) 13331343.

[47] Q. Zhao, et al., An NAC transcription factor orchestrates multiple features of cell wall development in Medicago truncatula, Plant J. 63 (2010) 100-114.

[48] B. Xu, et al., Contribution of NAC transcription factors to plant adaptation to land. Science 343 (2014) 1505-1508.

[49] R.L. McCarthy, R. Zhong, Z.-H. Ye, MYB83 is a direct target of SND1 and acts redundantly with MYB46 in the regulation of secondary cell wall biosynthesis in Arabidopsis, Plant Cell Physiol. 50 (2009) 1950-1964.

[50] J.H. Ko, W.C. Kim, K.H. Han, Ectopic expression of MYB46 identifies transcriptional regulatory genes involved in secondary wall biosynthesis in Arabidopsis, Plant J. 60 (2009) 64965.

[51] A. Patzlaff, et al., Characterisation of a pine MYB that regulates lignification, Plant J. 36 (2003a) 743-754.

[52] C. Bomal, et al., Involvement of Pinus taeda MYB1 and MYB8 in phenylpropanoid metabolism and secondary cell wall biogenesis: a comparative in planta analysis, J. Exp. Bot. 59 (2008) 3925-3939. 
[53] M. Goicoechea, et al., EgMYB2, a new transcriptional activator from Eucalyptus xylem, regulates secondary cell wall formation and lignin biosynthesis, Plant J. 43 (2005) 553-567.

[54] R.L. McCarthy, et al., The poplar MYB transcription factors, PtrMYB3 and PtrMYB20, are involved in the regulation of secondary wall biosynthesis, Plant Cell Physiol. 51 (2010) 10841090.

[55] R. Zhong, R.L. McCarthy, M. Haghighat, Z.-H. Ye, The poplar MYB master switches bind to the SMRE site and activate the secondary wall biosynthetic program during wood formation, PLoS One 8 (2013) e69219.

[56] R. Zhong, Z.-H. Ye, Transcriptional regulation of lignin biosynthesis, Plant Signal Behav. 4 (2009) 1028-1034.

[57] S. Steiner-Lange, et al., Disruption of Arabidopsis thaliana MYB26 results in male sterility due to non-dehiscent anthers. Plant J. 34 (2003) 519-528.

[58] C. Yang, et al., Arabidopsis MYB26/MALE STERILE35 regulates secondary thickening in the endothecium and is essential for anther dehiscence. Plant Cell 19 (2007) 534-548.

[59] D. Hatton, et al., Two classes of cis sequences contribute to tissue-specific expression of a PAL2 promoter in transgenic tobacco, Plant J. 7 (1995) 859-876.

[60] R. Zhong, Z.-H. Ye, MYB46 and MYB83 bind to the SMRE sites and directly activate a suite of transcription factors and secondary wall biosynthetic genes, Plant Cell Physiol. 53 (2012) 368-380.

[61] W.C. Kim, J.H. Ko, K.H. Han, Identification of a cis-acting regulatory motif recognized by MYB46, a master transcriptional regulator of secondary wall biosynthesis, Plant Mol. Biol. 78 (2012) 489-501.

[62] H. Cassan-Wang, et al., Identification of novel transcription factors regulating secondary cell wall formation in Arabidopsis, Front Plant Sci. 4 (2013) 189. 
[63] W.C. Kim, et al., MYB46 directly regulates the gene expression of secondary wallassociated cellulose synthases in Arabidopsis, Plant J. 73 (2013) 26-36.

[64] W.C. Kim, J.Y. Kim, J.H. Ko, H. Kang, K.H. Han, Identification of direct targets of transcription factor MYB46 provides insights into the transcriptional regulation of secondary wall biosynthesis. Plant Mol. Biol. 85 (2014) 589-599.

[65] S.G. Hussey, et al., SND2, a NAC transcription factor gene, regulates genes involved in secondary cell wall development in Arabidopsis fibres and increases fibre cell area in Eucalyptus, BMC Plant Biol. 11 (2011) 173.

[66] E.H. Grant, T. Fujino, E.P. Beers, A.M. Brunner, Characterization of NAC domain transcription factors implicated in control of vascular cell differentiation in Arabidopsis and Populus. Planta 232 (2010) 337-352.

[67] H.H. Wang, et al., Chimeric repressor of PtSND2 severely affects wood formation in transgenic Populus. Tree Physiol. 33 (2013) 878-886.

[68] D.M. Brown, L.A.H. Zeef, J. Ellis, R. Goodacreb, S.R. Turner, Identification of novel genes in Arabidopsis involved in secondary cell wall formation using expression profiling and reverse genetics. Plant Cell 17 (2005) 2281-2295.

[69] E. Li, et al., The Class II KNOX gene KNAT7 negatively regulates secondary wall formation in Arabidopsis and is functionally conserved in Populus, New Phytol. 194 (2012) 102115.

[70] E. Li, S. Wang, Y. Liu, J.G. Chen, C.J. Douglas OVATE FAMILY PROTEIN4 (OFP4) interaction with KNAT7 regulates secondary cell wall formation in Arabidopsis thaliana, Plant J. 67 (2011) 328-341. 
[71] A. Bhargava, et al., The interacting MYB75 and KNAT7 transcription factors modulate secondary cell wall deposition both in stems and seed coat in Arabidopsis, Planta 237 (2013) 1199-1211.

[72] M.C. Pomeranz, et al., The Arabidopsis tandem zinc finger protein AtTZF1 traffics between the nucleus and cytoplasmic foci and binds both DNA and RNA. Plant Physiol. 152 (2010) 151165.

[73] C. Zhao, U. Avci, E.H. Grant, C.H. Haigler, E.P. Beers, XND1, a member of the NAC domain family in Arabidopsis thaliana, negatively regulates lignocellulose synthesis and programmed cell death in xylem Plant J. 53 (2008) 425-436.

[74] J. Zhou, C. Lee, R. Zhong, Z.-H. Ye, MYB58 and MYB63 are transcriptional activators of the lignin biosynthetic pathway during secondary cell wall formation in Arabidopsis, Plant Cell 21 (2009) 248-266.

[75] S. Wang, et al., Regulation of secondary cell wall biosynthesis by poplar R2R3 MYB transcription factor PtrMYB152 in Arabidopsis. Sci. Rep. 4 (2014) 5054.

[76] D. Ohman, et al., MYB103 is required for FERULATE-5-HYDROXYLASE expression and syringyl lignin biosynthesis in Arabidopsis stems, Plant J. 73 (2012) 63-76.

[77] A. Kawaoka, et al., Functional analysis of tobacco LIM protein Ntlim1 involved in lignin biosynthesis, Plant J. 22 (2000) 289-301.

[78] Q. Zhao, et al., Syringyl lignin biosynthesis is directly regulated by a secondary cell wall master switch, Proc. Natl. Acad. Sci. USA 107 (2010) 14496-14501.

[79] J.M. Romano, et al., AtMYB61, an R2R3-MYB transcription factor, functions as a pleiotropic regulator via a small gene network. New Phytol. 195 (2012) 774-786. 
[80] L.J. Newman, D.E. Perazza, L. Juda, M.M. Campbell, Involvement of the R2R3-MYB, AtMYB61, in the ectopic lignification and dark-photomorphogenic components of the det3 mutant phenotype. Plant J. 37 (2004) 239-250.

[81] K. Hirano, et al., Identification of transcription factors involved in rice secondary cell wall formation. Plant Cell Physiol. 54 (2013) 1791-1802.

[82] H. Jin, et al., Transcriptional repression by AtMYB4 controls production of UV-protecting sunscreens in Arabidopsis, EMBO J. 19 (2000) 6150-6161.

[83] J. Preston, J. Wheeler, J. Heazlewood, S.F. Li, R.W. Parish, AtMYB32 is required for normal pollen development in Arabidopsis thaliana, Plant J. 40 (2004) 979-995.

[84] S. Legay, et al., Molecular characterization of $\operatorname{EgMYB1}$, a putative transcriptional repressor of the lignin biosynthetic pathway, Plant Sci. 173 (2007) 542-549.

[85] S. Legay, et al., EgMYB1, an R2R3 MYB transcription factor from eucalyptus negatively regulates secondary cell wall formation in Arabidopsis and poplar, New Phytol. 188 (2010) 774786.

[86] F.M. Sonbol, et al., The maize ZmMYB42 represses the phenylpropanoid pathway and affects the cell wall structure, composition and degradability in Arabidopsis thaliana, Plant Mol. Biol. 70 (2009) 283-296.

[87] S. Fornale, et al., ZmMYB31 directly represses maize lignin genes and redirects the phenylpropanoid metabolic flux, Plant J. 64 (2010) 633-644.

[88] H. Shen, et al., Functional characterization of the switchgrass (Panicum virgatum) R2R3MYB transcription factor PvMYB4 for improvement of lignocellulosic feedstocks, New Phytol. 193 (2012) 121-136. 
[89] Y.C. Lin, et al., SND1 transcription factor-directed quantitative functional hierarchical genetic regulatory network in wood formation in Populus trichocarpa. Plant Cell 25 (2013) 4324-4341.

[90] H. Aspeborg, et al., Carbohydrate-Active Enzymes Involved in the Secondary Cell Wall Biogenesis in Hybrid Aspen. Plant Physiol. 137 (2005) 983-997.

[91] U. Avci, H.E. Petzold, I.O. Ismail, E.P. Beers, C.H..Haigler, Cysteine proteases XCP1 and $\mathrm{XCP} 2$ aid micro-autolysis within the intact central vacuole during xylogenesis in Arabidopsis roots. Plant J. 56 (2008) 303-315.

[92] S. Farage-Barhom, S. Burd, L. Sonego, R. Perl-Treves, A. Lers, Expression analysis of the BFN1 nuclease gene promoter during senescence, abscission, and programmed cell death-related processes. J. Exp. Bot. 59 (2008) 3247-3258.

[93] C.L. Courtois-Moreau, et al., A unique program for cell death in xylem fibers of Populus stem. Plant J. 58 (2009) 260-274.

[94] A.R. Kulkarni, et al., The ability of land plants to synthesize glucuronoxylans predates the evolution of tracheophytes, Glycobiology 22 (2012) 439-451.

[95] T. Bennett, et al., SOMBRERO, BEARSKIN1, and BEARSKIN2 regulate root cap maturation in Arabidopsis. Plant Cell 22 (2010) 640-654.

[96] M. Fendrych, et al., Programmed cell death controlled by ANAC033/SOMBRERO determines root cap organ size in Arabidopsis. Curr. Biol. 24 (2014) 931-940.

[97] F. Yang, et al., Engineering secondary cell wall deposition in plants. Plant Biotechnol. J. 11 (2013) 325-335. 
Table 1. Transcription factors involved in regulating secondary wall biosynthesis

\begin{tabular}{|c|c|c|c|}
\hline Genes & Species & Functions & References \\
\hline \multicolumn{4}{|c|}{ Secondary wall NAC master switches (SWNs) } \\
\hline VND1 to VND7 & Arabidopsis & $\begin{array}{l}\text { Master switches activating secondary wall biosynthesis } \\
\text { and programmed cell death in vessels }\end{array}$ & {$[3,4]$} \\
\hline SND1, NST1 & Arabidopsis & $\begin{array}{l}\text { Master switches activating secondary wall biosynthesis in } \\
\text { fibers }\end{array}$ & {$[6-9]$} \\
\hline NST1, NST2 & Arabidopsis & $\begin{array}{l}\text { Master switches activating secondary wall biosynthesis in } \\
\text { anther endothecium }\end{array}$ & {$[5]$} \\
\hline PtrWNDs & $\begin{array}{l}\text { Populus } \\
\text { trichocarpa }\end{array}$ & $\begin{array}{l}\text { Master switches activating secondary wall biosynthesis } \\
\text { during wood formation }\end{array}$ & {$[33,41,43]$} \\
\hline EgWND1 & $\begin{array}{l}\text { Eucalyptus } \\
\text { gunnii }\end{array}$ & $\begin{array}{l}\text { Master switch activating secondary wall biosynthesis } \\
\text { during wood formation }\end{array}$ & {$[11]$} \\
\hline $\begin{array}{l}\text { PgNAC-4, } \\
\text { PgNAC-7 }\end{array}$ & $\begin{array}{l}\text { Picea glauca } \\
\text { (spruce) }\end{array}$ & Activate promoters of secondary wall biosynthesis genes & {$[44]$} \\
\hline OsSWNs & $\begin{array}{l}\text { Oryza sativa } \\
\text { (rice) }\end{array}$ & Master switch activating secondary wall biosynthesis & {$[38,45]$} \\
\hline ZmSWNs & $\begin{array}{l}\text { Zea mays } \\
\text { (maize) }\end{array}$ & Master switch activating secondary wall biosynthesis & {$[45]$} \\
\hline BdSWNs & $\begin{array}{l}\text { Brachypodium } \\
\text { distachyon }\end{array}$ & $\begin{array}{l}\text { Master switches activating secondary wall biosynthesis } \\
\text { and programmed cell death }\end{array}$ & {$[46]$} \\
\hline MtNST1 & $\begin{array}{l}\text { Medicago } \\
\text { truncatula }\end{array}$ & Activate secondary wall biosynthesis in fibers & {$[19]$} \\
\hline \multicolumn{4}{|c|}{ SWN activators and repressors } \\
\hline LBD18, LBD30 & Arabidopsis & $\begin{array}{l}\text { Positive feedback activation of } V N D 6 \text { and } V N D 7 \\
\text { in vessels }\end{array}$ & {$[35]$} \\
\hline VNI2 & Arabidopsis & Transcriptional repressor of $V N D$ s in vessels & {$[36]$} \\
\hline WRKY12 & Arabidopsis & $\begin{array}{l}\text { Transcriptional repressor of NST2 and secondary wall } \\
\text { thickening in pith parenchyma cells }\end{array}$ & {$[40]$} \\
\hline \multicolumn{4}{|c|}{ Secondary wall MYB master switches } \\
\hline $\begin{array}{l}\text { MYB46, } \\
\text { MYB83 }\end{array}$ & Arabidopsis & $\begin{array}{l}\text { Master switches activating secondary wall biosynthesis } \\
\text { in fibers and vessels }\end{array}$ & {$[13,49]$} \\
\hline $\begin{array}{l}\text { PtMYB4, } \\
\text { PtMYB8 }\end{array}$ & $\begin{array}{l}\text { Pinus taeda } \\
\text { (pine) }\end{array}$ & $\begin{array}{l}\text { MYB } 46 / 83 \text { orthologs; master switch activating secondary } \\
\text { wall biosynthesis }\end{array}$ & {$[11,51,52]$} \\
\hline EgMYB2 & $\begin{array}{l}\text { Eucalyptus } \\
\text { gunnii }\end{array}$ & $\begin{array}{l}\text { MYB } 46 / 83 \text { orthologs; master switch activating secondary } \\
\text { wall biosynthesis }\end{array}$ & {$[11,53]$} \\
\hline $\begin{array}{l}\text { PtrMYB2, } \\
\text { PtrMYB3, } \\
\text { PtrMYB20, } \\
\text { PtrMYB21 }\end{array}$ & $\begin{array}{l}\text { Populus } \\
\text { trichocarpa }\end{array}$ & $\begin{array}{l}\text { MYB46/83 orthologs; master switch activating secondary } \\
\text { wall biosynthesis during wood formation }\end{array}$ & {$[54,55]$} \\
\hline OsMYB46 & $\begin{array}{l}\text { Oryza sativa } \\
\text { (rice) }\end{array}$ & $\begin{array}{l}\text { MYB } 46 / 83 \text { orthologs; Master switches activating secondar } \\
\text { biosynthesis }\end{array}$ & {$[45]$} \\
\hline ZmMYB46 & $\begin{array}{l}\text { Zea mays } \\
\text { (maize) }\end{array}$ & Master switches activating secondary wall biosynthesis & {$[45]$} \\
\hline MYB26 & Arabidopsis & $\begin{array}{l}\text { Master switches activating secondary wall biosynthesis } \\
\text { in anther endothecium }\end{array}$ & {$[57,58]$} \\
\hline \multicolumn{4}{|c|}{ Downstream transcription factors activated by secondary wall NAC and MYB master switches } \\
\hline SND2, SND3 & Arabidopsis & $\begin{array}{l}\text { Their dominant repression results in reduced secondary } \\
\text { wall thickening in fibers }\end{array}$ & [14] \\
\hline PtrSND2 & Populus & Its dominant repression results in reduced secondary & $\begin{array}{l}67] \\
\end{array}$ \\
\hline
\end{tabular}




\begin{tabular}{|c|c|c|c|}
\hline & trichocarpa & wall thickening in fibers & \\
\hline KNAT7 & Arabidopsis & $\begin{array}{l}\text { Its mutation results in collapsed vessels but increased wall } \\
\text { thickening in fibers }\end{array}$ & {$[14,68,69]$} \\
\hline XND1 & Arabidopsis & $\begin{array}{l}\text { Its overexpression results in inhibition of secondary wall } \\
\text { thickening and programmed cell death in vessels }\end{array}$ & [73] \\
\hline $\begin{array}{l}\text { MYB52, MYB54 } \\
\text { MYB69 }\end{array}$ & Arabidopsis & $\begin{array}{l}\text { Their dominant repression results in reduced secondary } \\
\text { wall thickening in fibers }\end{array}$ & [14] \\
\hline MYB103 & Arabidopsis & $\begin{array}{l}\text { Its dominant repression or mutation results in reduced } \\
\text { secondary wall thickening and syringyl lignin }\end{array}$ & {$[14,76]$} \\
\hline \multicolumn{4}{|c|}{ Lignin-specific activators } \\
\hline MYB58, MYB63 & Arabidopsis & Transcriptional activators of lignin biosynthesis & [74] \\
\hline PtrMYB28 & $\begin{array}{l}\text { Populus } \\
\text { trichocarpa }\end{array}$ & $\begin{array}{l}\text { MYB58/63 ortholog; activator of lignin biosynthesis } \\
\text { genes }\end{array}$ & [56] \\
\hline MYB85 & Arabidopsis & Transcriptional activator of lignin biosynthesis & [14] \\
\hline PtMYB1 & $\begin{array}{l}\text { Pinus taeda } \\
\text { (pine) }\end{array}$ & $\begin{array}{l}\text { MYB85 ottholog; transcriptional activator of lignin } \\
\text { biosynthesis }\end{array}$ & [52] \\
\hline PtrMYB152 & $\begin{array}{l}\text { Populus } \\
\text { trichocarpa }\end{array}$ & $\begin{array}{l}\text { MYB43 ortholog; Transcriptional activator of lignin } \\
\text { biosynthesis }\end{array}$ & [75] \\
\hline NtLIM1 & $\begin{array}{l}\text { Nicotiana } \\
\text { tabacum }\end{array}$ & $\begin{array}{l}\text { Transcriptional activator of lignin } \\
\text { biosynthesis }\end{array}$ & [77] \\
\hline BLH6 & Arabidopsis & Its mutation results in reduced lignin deposition & [62] \\
\hline \multicolumn{4}{|c|}{ Lignin-specific repressors } \\
\hline $\begin{array}{l}\text { MYB4, MYB7, } \\
\text { MYB32 }\end{array}$ & Arabidopsis & Transcriptional repressors of lignin biosynthesis & {$[82,83]$} \\
\hline $\begin{array}{l}\text { ZmMYB31, } \\
\text { ZmMYB42 }\end{array}$ & $\begin{array}{l}\text { Zea mays } \\
\text { (maize) }\end{array}$ & $\begin{array}{l}\text { MYB4 orthologs; transcriptional repressors of lignin } \\
\text { biosynthesis }\end{array}$ & {$[86,87]$} \\
\hline PvMYB4 & $\begin{array}{l}\text { Panicum } \\
\text { Virgatum } \\
\text { (switchgrass) }\end{array}$ & $\begin{array}{l}\text { MYB4 ortholog; transcriptional repressor of lignin } \\
\text { biosynthesis }\end{array}$ & [88] \\
\hline EgMYB1 & $\begin{array}{l}\text { Eucalyptus } \\
\text { gunnii }\end{array}$ & $\begin{array}{l}\text { MYB4 ortholog; transcriptional repressor of lignin } \\
\text { biosynthesis }\end{array}$ & {$[84,85]$} \\
\hline $\begin{array}{l}\text { MYB52, HB5, } \\
\text { HB15, } \\
\text { At3g11280 }\end{array}$ & Arabidopsis & $\begin{array}{l}\text { Negative regulators of lignin biosynthesis; their mutations } \\
\text { result in hyperlignified secondary walls }\end{array}$ & [62] \\
\hline
\end{tabular}

\section{Figure Legends}


Fig. 1. Diagram of the transcriptional network regulating secondary wall biosynthesis. The network employs a multi-leveled feed-forward loop regulatory structure, in which the top-level secondary wall NAC master switches directly activate the second-level secondary wall MYB master switches, both of which further activate downstream transcription factors and secondary wall biosynthesis genes via binding to the SNBE and SMRE sites in their promoters, respectively. It is proposed that the concerted actions of secondary wall NAC master switches, secondary wall MYB master switches and their downstream transcription factors activate the secondary wall biosynthesis genes, which leads to biosynthesis and deposition of cellulose, xylan and lignin in secondary wall-forming cells. Regulation of lignin biosynthesis involves ligninspecific transcriptional activators and repressors. Secondary wall NAC master switches also directly regulate genes involved in programmed cell death. Only some representative transcription factors that have been proven to be involved in regulation of secondary wall biosynthesis are included in the diagram.

Fig. 2. Regulation of secondary wall biosynthesis by secondary wall NAC and secondary wall MYB master switches in Arabidopsis. (A) Phloroglucinol-HCl staining of a cross section of wild-type stem showing thick lignified secondary walls in interfascicular fibers (if), xylary fibers (xf) and vessels (ve). (B) Phloroglucinol-HCl staining of a stem cross section showing that simultaneous RNA interference (RNAi) of SND1 and NST1 expression results in a loss of secondary wall thickening in interfascicular fibers and xylary fibers but not in vessels. (C) Toluidine blue-staining of a stem cross section showing that simultaneous RNAi inhibition of MYB46 and MYB83 causes a loss of secondary wall thickening in interfascicular fibers and xylary fibers and a severe deformation of vessel morphology. Inset in (C) is a close-up xylem bundle displaying the collapsed vessel phenotype (Arrows). (D) to (F) Leaf epidermis of wild- 
type (D), SND1 overexpressor (SND-OE) and MYB46 overexpressor (MYB46-OE). Note the ectopic deposition of reticulated secondary walls in the epidermal cells induced by overexpression of SND1 (E) and MYB46 (F).

Fig. 3. Identification of the SNBE and SMRE sites in the promoters of secondary wallassociated transcription factors and secondary wall biosynthesis genes. Both the forward and reverse strands of the $1.5-\mathrm{kb}$ promoter sequences were examined for the presence of the SNBE and SMRE sites based on the SNBE consensus sequence $[(\mathrm{T} / \mathrm{A}) \mathrm{NN}(\mathrm{C} / \mathrm{T})(\mathrm{T} / \mathrm{C} / \mathrm{G}) \mathrm{TNNNNNNNA}(\mathrm{A} / \mathrm{C}) \mathrm{GN}(\mathrm{A} / \mathrm{C} / \mathrm{T})(\mathrm{A} / \mathrm{T})]$ and the SMRE consensus sequence $[\mathrm{ACC}(\mathrm{A} / \mathrm{T}) \mathrm{A}(\mathrm{A} / \mathrm{C})(\mathrm{T} / \mathrm{C})]$.

Fig. 4. Phylogenetic relationship of secondary wall-associated NAC domain transcription factors. Only those NACs that have been shown to be involved in regulation of secondary wall biosynthesis are selected for constructing the phylogenetic tree using the neighbor-joining algorithm. Bootstrap values are shown in percentages at the nodes. The 0.1 scale denotes $10 \%$ change. NAC sequences are from Arabidopsis thaliana, rice (Oryza sativa; Os), maize (Zea mays; Zm), Brachypodium distachyon (Bd), Medicago truncatula (Mt), soybean (Glycine max; Gm), poplar (Populus trichocarpa; Ptr), and Eucalyptus gunnii (Eg). Each gene name is followed by its GenBank accession number or gene locus identifier.

Fig. 5. Phylogenetic relationship of secondary wall-associated MYB transcription factors. Only those MYBs that have been shown to be involved in regulation of secondary wall biosynthesis are selected for constructing the phylogenetic tree using the neighbor-joining algorithm. Bootstrap values are shown in percentages at the nodes. The 0.1 scale denotes $10 \%$ change. 
MYB sequences are from Arabidopsis thaliana, rice (Oryza sativa; Os), maize (Zea mays; Zm), poplar (Populus trichocarpa; Ptr), Eucalyptus gunnii (Eg), and pine (Pinus taeda; Pt). Each gene name is followed by its GenBank accession number or gene locus identifier. 


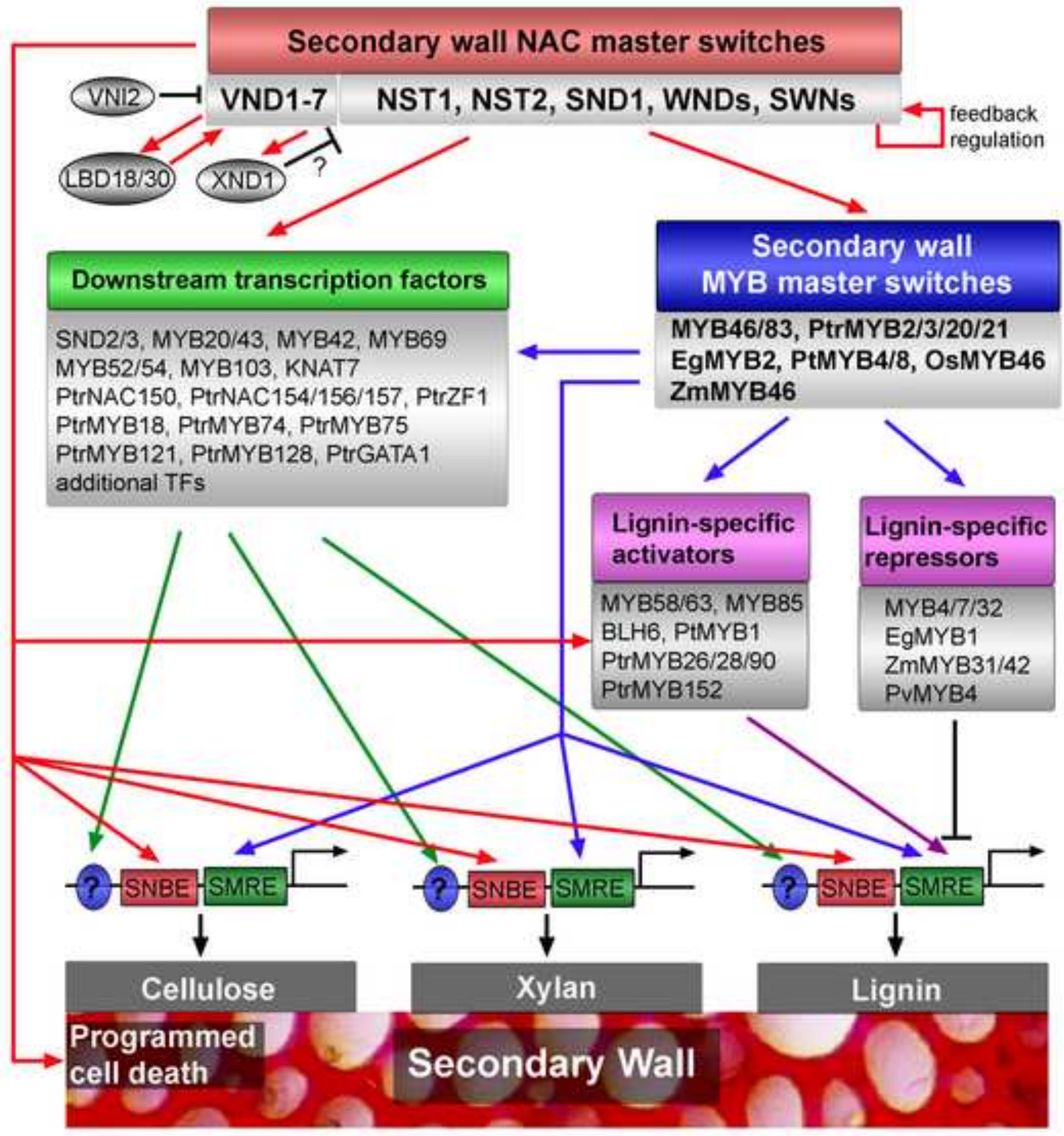



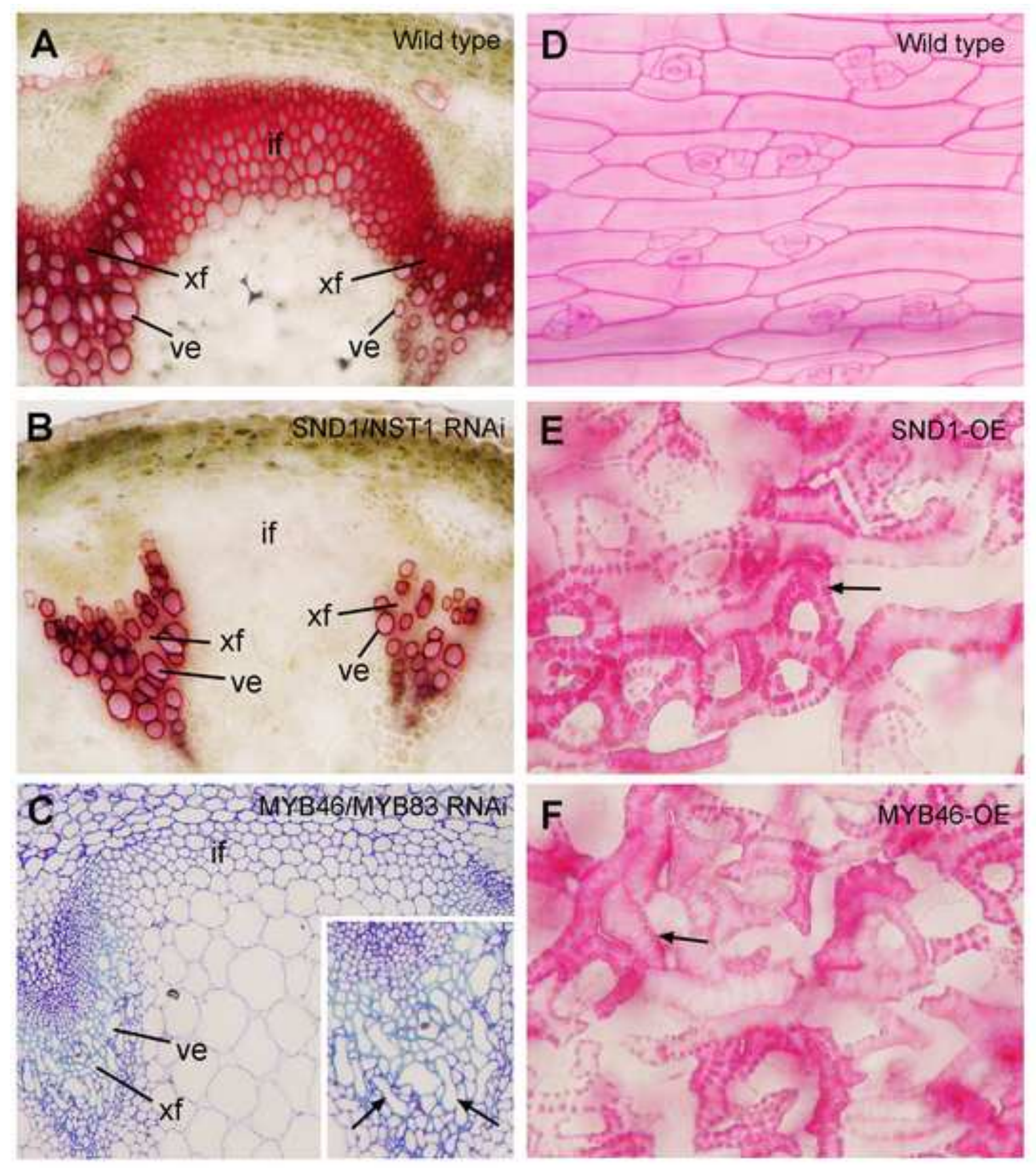

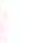

.

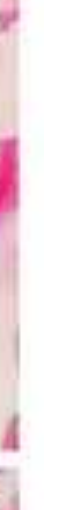

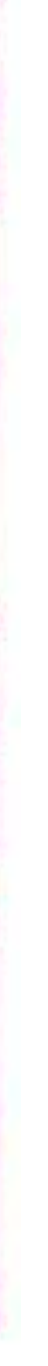




\begin{tabular}{|c|c|c|c|}
\hline Gene & KGI code & $\begin{array}{l}\text { Number of } \\
\text { SVBE, dites }\end{array}$ & $\begin{array}{l}\text { Number of } \\
\text { SMIRE sites: }\end{array}$ \\
\hline \multicolumn{4}{|c|}{ Transcription facton: } \\
\hline VNDI & $A 12 g 18060$ & 3 & 1 \\
\hline VND2 & A14g36160 & 5 & 5 \\
\hline VND3 & At5g66 300 & 1 & 1 \\
\hline VND4 & Attg 12260 & 4 & 2 \\
\hline VNDS & Allg62700 & 2 & 9 \\
\hline VND6: & Aisg62380 & 0 & 4 \\
\hline VND7 & Alig71930 & 2 & 1 \\
\hline NSTI & As2 46770 & 3 & 1 \\
\hline $\mathrm{NST} 2$ & At3g61910 & 1 & 0 \\
\hline SNDI & Atg 32770 & 1 & 1 \\
\hline SND2 & $\mathrm{A} 14 \mathrm{~g} 28500$ & 4 & I \\
\hline SND3 & Atg 28470 & 10 & 4 \\
\hline MYH 46 & Assg 12870 & 10 & 0 \\
\hline MYR83 & At3goxs00 & II & 2 \\
\hline MYE20 & Atlg66230 & 3 & 1 \\
\hline $\mathrm{MYB4} 2$ & A14g12350 & 5 & 4 \\
\hline MYB43 & A15gl6600 & 2 & 5 \\
\hline MYBS2: & Ailg 17950 & 2 & 5 \\
\hline MYits4 & A11g 23410 & 5 & 2 \\
\hline MYB58. & Allg16490 & 2 & 4 \\
\hline MYH63 & Aitg79180 & 2 & 8 \\
\hline MYH69 & A14833450 & 1 & 4 \\
\hline MYBS5 & Ar4g22650 & 2 & 1 \\
\hline MYB 103 & AdIg63910 & 4 & 1 \\
\hline KNAT7 & Allg62090 & 3 & 3 \\
\hline LOBI5 & $A 120+0470$ & 6 & 4 \\
\hline LOB 30 & $\mathrm{~A} 14 \mathrm{~g} O 0220$ & 3 & 3 \\
\hline XNDI & A15g64530 & 2 & 2 \\
\hline MYH & Al4g 38620 & 4 & 8 \\
\hline MYมz & A12g 16720 & 3 & 2 \\
\hline MYB32 & At4g34990 & 5 & 8 \\
\hline B. 116 : & A 4934610 & 9 & 4 \\
\hline \multicolumn{4}{|c|}{ Cellulone biosynthetic genes] } \\
\hline $\operatorname{Ces} A_{4}$ & At5g 44030 & 1 & 6 \\
\hline CesA7 & Alsg17420 & 5 & 3 \\
\hline CesA8 & At+g18780 & 4 & 4 \\
\hline
\end{tabular}

\begin{tabular}{|c|c|c|c|}
\hline Geae & AGI code & $\begin{array}{l}\text { Number of } \\
\text { SNHE sites }\end{array}$ & $\begin{array}{l}\text { Number of } \\
\text { SMRE sites }\end{array}$ \\
\hline \multicolumn{4}{|c|}{ Xylan blosynthetic genes: } \\
\hline IRXY & Ang 37090 & 2 & 2 \\
\hline 194ARX9: & $\mathrm{AtI}_{2} 27600$ & 5 & 1 \\
\hline $\operatorname{IR} \times 14$ & $A 1436890$ & 6 & 2 \\
\hline 114H/RXI41. & A 5,67240 & 6 & 1 \\
\hline IRX8 & AL5 54690 & 7 & 4 \\
\hline PARVUS & Atig10300 & 7 & 1 \\
\hline FRAB & $\mathrm{AL}_{2} 28110$ & 5 & $\$$ \\
\hline $\mathrm{FrH}$ & $\mathrm{ABS}_{\mathrm{S}} 22940$ & 2 & 2 \\
\hline IRX10kGUT2 & $\mathrm{AtI}_{\mathrm{g}} 27440$ & 7 & 3 \\
\hline IRXIOLGUTI & $\mathrm{AL}_{6} 61840$ & 3 & 0 \\
\hline GUXI & $\mathrm{AB}_{5} 18000$ & 7 & 3 \\
\hline GUX2 & Atte33330 & 6 & 3 \\
\hline $\mathrm{GUX3}$ & AtIe77130 & 0 & 2 \\
\hline GXMi & Atl g09610 & 4 & 2 \\
\hline GXM2 & $\mathrm{At} \mathrm{g}_{\mathrm{g}} 099 \mathrm{OS}$ & 3 & 4 \\
\hline GXM3/GXMT1 & Atig 33800 & 7 & 1 \\
\hline IRX15 & $A+3,50220$ & 4 & 1 \\
\hline IRXISt. & A 15867210 & 5 & 5 \\
\hline ЕSKL/TВ1 29 & $\mathrm{A1} 3 \mathrm{z} 55990$ & 8 & 6 \\
\hline RWAI & At 5.46340 & 6 & 4 \\
\hline RWA2 & $\mathrm{AL}_{8} 06550$ & 6 & 1 \\
\hline RWA3 & $A Z_{g} 34410$ & 2 & 4 \\
\hline RWA & $\mathrm{All}_{2} 29890$ & 3 & 7 \\
\hline \multicolumn{4}{|c|}{ L.tgnin biosyntbetic genes: } \\
\hline PALI & $\mathrm{AL} 2 \mathrm{~s}, 37040$ & 5 & 6 \\
\hline PAL2 & $A B_{g} 53260$ & 3 & 9 \\
\hline PAL.3 & $\mathrm{AL}_{5} 04230$ & 2 & 3 \\
\hline $\mathrm{C} 4 \mathrm{H}$ & Aceg30490 & 2 & 6 \\
\hline $4 C 1.1$ & Atlg51680 & 6 & 8 \\
\hline 467.2 & $A B_{g} 21240$ & 1 & 5 \\
\hline $\mathrm{HCT}$ & At 5 s 48930 & 3 & 7 \\
\hline C3HII & $\mathrm{At} 2_{5} 40890$ & 3 & 5 \\
\hline CCoAOMTI & Alig 34050 & 3 & 4 \\
\hline CCRI & At1: 15950 & 2 & 3 \\
\hline F5H1 & Altg 36220 & 3 & 3 \\
\hline COMT & Atsg54160 & $i$ & 3 \\
\hline CAD5 & $\mathrm{At} 4 \mathrm{~g}, 34230$ & 7 & 3 \\
\hline CADS & Attg37970 & 1 & 4 \\
\hline LAC4 & $\mathrm{AL} 2 \mathrm{~g} 3080$ & 4 & 4 \\
\hline I.ACH & Ats 603260 & 4 & 3 \\
\hline L.MC12 & $A B S_{5} 05390$ & 8 & 2 \\
\hline IACI? & $A L 560020$ & 8 & 3 \\
\hline Peroxidase 72 & At5ig66390 & 2 & 2 \\
\hline PDRL/ALACOC29 & ABg16340 & $i$ & 6 \\
\hline
\end{tabular}


Figure 5
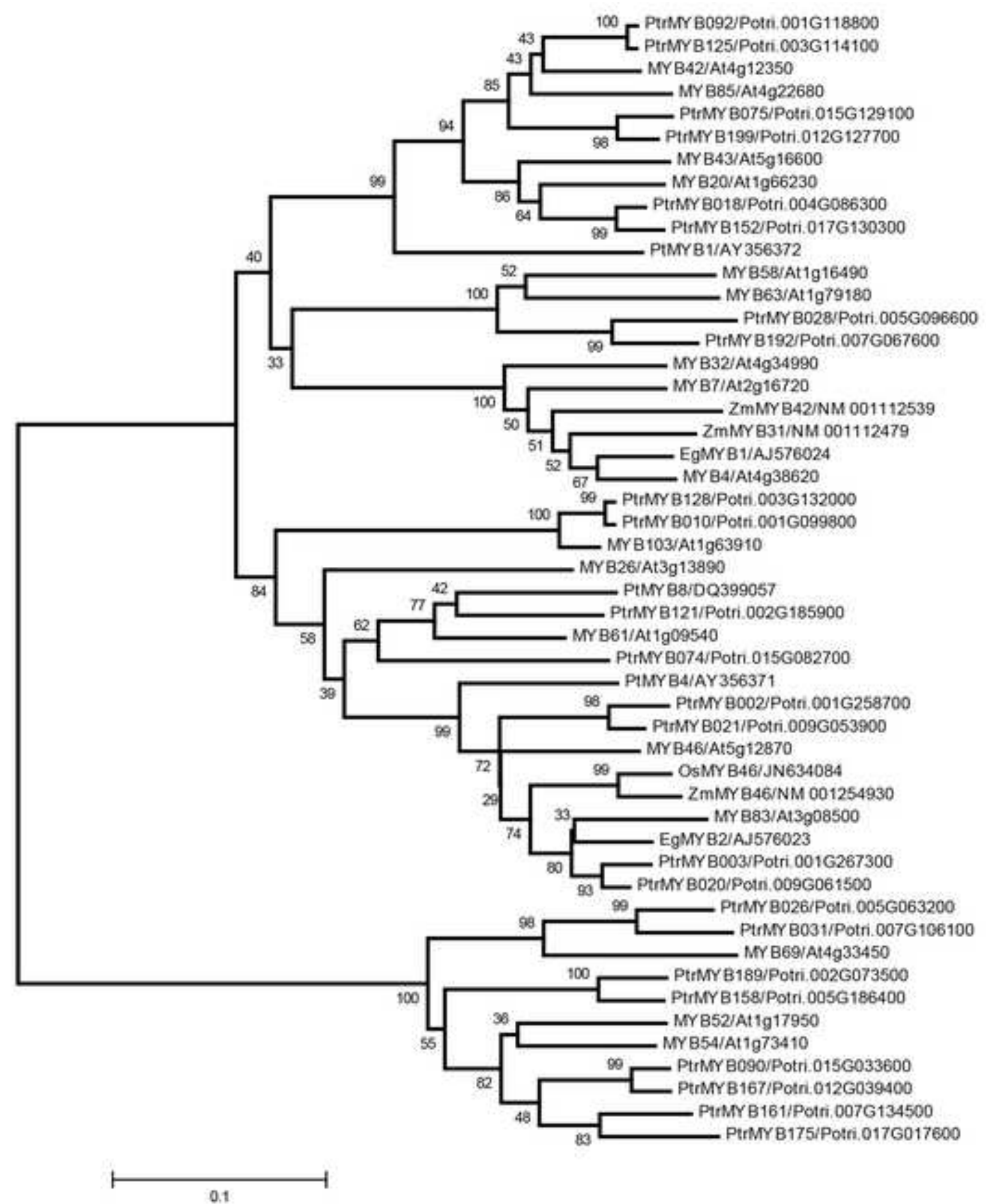

- MY B85iAt4g22680

PtrMYB1991

MYB43/At5g 16600

trMY B018/Potri 004G086300

MYB5B/At1g16490

- PtrMYB028/Potri. 0056096600

(8)

YY32/At4g34990

- ZmMYB42/NM 001112539

EgMYB1/AJ576024

MY B4/At4g38620

99 - PtrMY B128/Potri 0036132000

L PtrMY B010/Potri.001G099800 